UMHEP-414

\title{
Two-loop Analysis of Vector Current Propagators in Chiral Perturbation Theory
}

\author{
Eugene Golowich and Joachim Kambor 円 \\ Department of Physics and Astronomy \\ University of Massachusetts \\ Amherst MA 01003 USA
}

\begin{abstract}
We perform a calculation of the isospin and hypercharge vector current propagators $\left(\Delta_{\mathrm{V} 33}^{\mu \nu}\left(q^{2}\right)\right.$ and $\left.\Delta_{\mathrm{V} 88}^{\mu \nu}\left(q^{2}\right)\right)$ to two loops in chiral perturbation theory. The analysis is carried out with straightforward Feynman diagram methods by making appropriate use of external vector sources. Counterterms from the $\mathcal{O}\left(q^{6}\right)$ chiral lagrangian, required to absorb divergences and scale dependence encountered at the two-loop level, are constructed. Our final results are finite, covariant, and scaleindependent. Several applications are described, including a comparison of the two-loop isospin vector spectral function with data and the construction of new chiral sum rules.
\end{abstract}

\footnotetext{
${ }^{1}$ address after September 1, 1994: Division de Physique Théorique, Institut de Physique Nucléaire, F-91406 Orsay Cedex, France
} 


\section{Introduction}

A decade ago, Gasser and Leutwyler performed a general one-loop analysis of chiral perturbation theory. 11], Among their results were representations of the isospin vector current and axialvector current propagators, valid up to $\mathcal{O}\left(q^{4}\right)$ corrections. In this paper, we shall describe a calculation to two-loop order in chiral perturbation theoryt (hereafter ChPT) of both the isospin and hypercharge vector current propagators, yielding representations valid up to $\mathcal{O}\left(q^{6}\right)$ corrections.

In the years following the publication of the Gasser-Leutwyler papers cited above, there appeared a dramatic increase in the number of studies utilizing ChPT to analyze hadrons at low energies. However, most of these involved either tree-level or one-loop calculations. In fact, there currently exists a need for higher-order calculations which meaningfully probe the convergence properties of ChPT. This forms an important impetus for performing our calculation.

There is a more phenomenologically-oriented way of stating our motivation which actually bears on future applications of our work - the subject of chiral sum rules. It has long been known that among the predictions of chiral symmetry are the following set of sum rules,

$$
\begin{aligned}
& \int_{0}^{\infty} d s \frac{\rho_{\mathrm{V}}(s)-\rho_{\mathrm{A}}(s)}{s}=-4 L_{10}^{r}\left(M_{K}^{2}\right)+\frac{1}{48 \pi^{2}} \ln \frac{M_{K}^{2}}{M_{\pi}^{2}}-\frac{1}{32 \pi^{2}}, \\
& \int_{0}^{\infty} d s\left(\rho_{\mathrm{V}}(s)-\rho_{\mathrm{A}}(s)\right)=F_{\pi}^{2}, \\
& \int_{0}^{\infty} d s s\left(\rho_{\mathrm{V}}(s)-\rho_{\mathrm{A}}(s)\right)=0, \\
& \int_{0}^{\infty} d s s \ln \left(\frac{s}{\Lambda^{2}}\right)\left(\rho_{\mathrm{V}}(s)-\rho_{\mathrm{A}}(s)\right)=-\frac{16 \pi^{2} F_{\pi}^{2}}{3 e^{2}}\left(m_{\pi^{ \pm}}^{2}-m_{\pi^{0}}^{2}\right) .
\end{aligned}
$$

In the first of these 4 , the quantity $L_{10}^{r}\left(M_{K}^{2}\right)$ is the renormalized coefficient of an $\mathcal{O}\left(E^{4}\right)$ counterterm operator appearing in the $S U(3)$ chiral lagrangian of $Q C D$. We do not comment further on this quantity here insofar as much of Sect. 4 and Sect. 5 is devoted to a study of counterterms and their renormalization. The next two relations are respectively the first and second Weinberg sum rules. 5 The final sum rule is a formula for the $\pi^{ \pm}-\pi^{0}$ mass

\footnotetext{
${ }^{2}$ We stress for clarity's sake that our analysis throughout will be in the standard ChPT and not the so-called generalized ChPT of Ref. [3].
} 
splitting in the chiral limit. 6. Although apparently containing an arbitrary energy scale $\Lambda$, this sum rule is actually independent of $\Lambda$ by virtue of the preceding relation.

Although the above sum rules were derived almost 30 years ago, a full phenomenological test of their content was not carried out until recently []. In principle, the underlying strategy employed in Ref. [7] was to divide the energy region into three intervals as follows. In the large energy region, from $s \rightarrow \infty$ down to energies where data first become available, operator product expansion methods were used. At intermediate energies, the vector and axialvector spectral functions $\rho_{\mathrm{V}}(s)$ and $\rho_{\mathrm{A}}(s)$ were extracted from tau decay distributions and $e^{+} e^{-} \rightarrow 2 \pi, 4 \pi$ cross sections. Finally, the threshold behavior $\left(s \rightarrow 4 m_{\pi}^{2}\right.$ ) of the spectral functions was constrained by means of chiral symmetry. In practice, however, this final step was not found to be crucial to the calculation and as a result was not implemented. The analysis of Ref. [7] ultimately led to a successful fit to all four chiral sum rules, which is highly nontrivial in view of the strong distortions experienced by the spectral functions from the various moments.

Let us study the threshold prediction of ChPT for the isospin spectral function. The dashed curve in Fig. 1 is the one-loop prediction of Gasser and Leutwyler and is to be compared with data taken from $e^{+} e^{-} \rightarrow \pi^{+} \pi^{-}$ scattering. One readily sees that it does not take long for the one-loop prediction to disagree with experiment as the energy is increased above the two-pion threshold. This is one of several reasons for computing to the next order of ChPT.

We conclude this section with an outline of the rest of the paper. Section 2 contains a discussion of our overall strategy for carrying out the calculation. The lowest-order lagrangian $\mathcal{L}^{(2)}$ is used to illustrate certain aspects of the discussion. Section 3 summarizes both the one-loop and two-loop chiral analysis and introduces $\mathcal{L}^{(4)}$, the $\mathcal{O}\left(q^{4}\right)$ chiral lagrangian, to the calculation. Section 4 covers the counterterm analysis, reviewing the presence of the $\mathcal{O}\left(q^{4}\right)$ counterterms and especially generating a complete list of $\mathcal{O}\left(q^{6}\right)$ counterterms relevant for our purposes. Section 5 contains a statement of our renormalization procedure. The use of the renormalization group (RG hereafter) to determine the scale dependence of various counterterms is discussed and the RG equations are solved to the needed order. Section 6 contains the explicit representations for the isospin and hypercharge vector current propagators which result from our calculation. Section 7 contains two applications of our results. The improved isospin vector spectral func- 
tion, now containing next-to-leading order contributions, is again compared with data. In addition, the asymptotic behaviour of the vector-propagators is employed to derive a new set of chiral sum rules. We conclude the main body of the paper in Section 8 by summarizing our findings and proposing further research on the subject. There are also two Appendices, the first devoted to defining the $d$-dimensional integrals which occur in our calculation and the second to giving an independent determination of the isospin spectral function $\rho_{\mathrm{V} 33}(s)$ at next-to-leading order.

\section{Procedure}

Consider the $S U(3)$ vector currents, 9

$$
V_{a}^{\mu}=\bar{q} \frac{\lambda_{a}}{2} \gamma^{\mu} q
$$

where $a=1, \ldots, 8$ and $q=(u d s)$. For the cases $a=b=3$ and $a=b=8$,

the corresponding two-current time-ordered products can be expressed as 10

$$
\begin{aligned}
& \left\langle 0\left|T\left(V_{a}^{\mu}(x) V_{b}^{\nu}(0)\right)\right| 0\right\rangle= \\
& \quad i \int_{0}^{\infty} d s \rho_{\mathrm{V} a b}(s)\left(\square g^{\mu \nu}-\partial^{\mu} \partial^{\nu}\right) \int \frac{d^{4} p}{(2 \pi)^{4}} \frac{e^{-i p \cdot x}}{p^{2}-s+i \epsilon},
\end{aligned}
$$

where the quantities $\rho_{\mathrm{V} a b}(s)$ are the spin-one vector spectral functions. The vector current propagators are Fourier transforms of the spatial two-point functions,

$$
\Delta_{V a b}^{\mu \nu}\left(q^{2}\right) \equiv i \int d^{4} x e^{i q \cdot x}\left\langle 0\left|T\left(V_{a}^{\mu}(x) V_{b}^{\nu}(0)\right)\right| 0\right\rangle
$$

In this paper, we shall use ChPT to calculate the low energy behavior of the isospin and hypercharge vector-current propagators $\Delta_{V 33}^{\mu \nu}\left(q^{2}\right)$ and $\Delta_{V 88}^{\mu \nu}\left(q^{2}\right)$. Since the underlying current is conserved for each of these, we have the tensor decomposition

$$
\Delta_{V a b}^{\mu \nu}\left(q^{2}\right)=\left(q^{\mu} q^{\nu}-q^{2} g^{\mu \nu}\right) \Pi_{a b}\left(q^{2}\right) \quad(a=b=3,8) .
$$

We shall refer to this tensor structure as covariant. In the course of calculating $\Delta_{V 33}^{\mu \nu}\left(q^{2}\right)$ and $\Delta_{V 88}^{\mu \nu}\left(q^{2}\right)$, one encounters a different class of contributions which are proportional to $g^{\mu \nu}$. We call these terms noncovariant. Of course, the set of noncovariant terms must ultimately cancel. 


\section{External Vector Sources}

In accordance with the plan to carry out a general analysis of ChPT to one-loop, Gasser and Leutwyler employed the so-called background-field method.11 This had the advantage of generating all possible $\mathcal{O}\left(q^{4}\right)$ counterterms. However, since our attention will be strictly limited to the conserved vector-current propagators $\Pi_{33}$ and $\Pi_{88}$, we have adopted a rather different approach. We observe that it is sufficient to compute all Feynman diagrams of the type depicted in Fig. 2, i.e. those with a single incoming external vector source undergoing a transition to a single outgoing external vector source. It will become clear that this method is both accessible and straightforward to implement.

Let us illustrate the procedure in terms of the lowest-order chiral lagrangian $\mathcal{L}^{(2)}$,

$$
\mathcal{L}^{(2)}=\frac{F_{0}^{2}}{4} \operatorname{Tr}\left(D_{\mu} U D^{\mu} U^{\dagger}\right)+\frac{F_{0}^{2}}{4} \operatorname{Tr}\left(\chi U^{\dagger}+U \chi^{\dagger}\right) .
$$

In the above, $F_{0}$ is the pseudoscalar meson decay constant to lowest order and $\chi=2 B_{0} \mathbf{m}$ is proportional to the quark mass matrix with

$$
B_{0}=\frac{m_{\pi}^{2}}{2 \hat{m}}=\frac{m_{K}^{2}}{\hat{m}+m_{s}}=\frac{3 m_{\eta}^{2}}{2\left(\hat{m}+2 m_{s}\right)} .
$$

Note that we work in the isospin symmetric limit of $m_{u}=m_{d} \equiv \hat{m}$. The field variable $U$ is defined in terms of the pseudoscalar meson fields $\left\{\phi_{a}\right\}$,

$$
U \equiv \exp \left(i \lambda_{a} \cdot \phi_{a} / F_{0}\right)
$$

and we construct the covariant derivative $D_{\mu} U$ in terms of external vector sources $v_{\mu}$,

$$
D_{\mu} U \equiv \partial_{\mu} U+i v_{\mu} U-i U v_{\mu} .
$$

The vector source $v_{\mu}$ has a component $v_{\mu}^{a}$ for each of the $S U(3)$ flavors,

$$
v_{\mu} \equiv \frac{\lambda_{a}}{2} \cdot v_{\mu}^{a}
$$

Suppose that we use the above form of $\mathcal{L}^{(2)}$ to compute a Feynman diagram of the type in Fig. 2. For now, consider a term in $\mathcal{L}^{(2)}$ which is linear in the vector source $v_{\mu}$,

$$
\mathcal{L}_{\mathrm{int}}=-v_{\mu}^{c}(x) V_{c}^{\mu}(x)
$$


The quantity $V_{c}^{\mu}(x)$ which is coupled to the external source $v_{\mu}^{c}$ must, by construction, be the corresponding vector current. To calculate the given Feynman diagram, we must work with the second-order operator $\mathcal{S}_{2}$,

$$
\mathcal{S}_{2}-1=\frac{i^{2}}{2 !} \int d^{4} x d^{4} y T\left(\mathcal{L}_{\text {int }}(x) \mathcal{L}_{\text {int }}(y)\right)
$$

Upon taking the matrix element for the diagram in Fig. 2 and employing translation invariance, we easily obtain the result,

$$
\left\langle f\left|\mathcal{S}_{2}-1\right| i\right\rangle=i(2 \pi)^{4} \delta^{(4)}\left(q^{\prime}-q\right) \epsilon_{\mu}^{\dagger}\left(q^{\prime}\right) \mathcal{M}_{a b}^{\mu \nu} \epsilon_{\nu}(q)
$$

where the invariant amplitude $\mathcal{M}_{a b}^{\mu \nu}$ is found to be

$$
\mathcal{M}_{a b}^{\mu \nu}=i \int d^{4} x e^{i q \cdot x}\langle 0| T\left(V_{a}^{\mu}(x) V_{b}^{\nu}(0)|0\rangle \equiv \Delta_{V a b}^{\mu \nu}\left(q^{2}\right)\right.
$$

That is, one just computes the invariant amplitudes for the class of diagrams in Fig. 2 and the result is the vector-current propagator of the relevant flavor. The only complication is that amplitudes corresponding to the linear interaction of Eq. (14) are generally noncovariant and one must thus include contact terms which are bilinear in the external vector sources. For example, in $S U(3)$ notation the full interaction induced by the chiral lagrangian $\mathcal{L}^{(2)}$ is

$$
\mathcal{L}_{\text {int }}=-f_{a b c} \phi_{b} \partial^{\mu} \phi_{c} v_{\mu}^{a}+\frac{1}{2} f_{a d g} f_{b c g} \phi_{c} \phi_{d} v_{a}^{\mu} v_{b}^{\nu},
$$

where the two terms are to be calculated respectively in second and first order perturbation theory. In the next section, we shall use this lowestorder interaction to begin our treatment of the vector-current propagators to one-loop order.

To recap briefly, our calculational program is to use isospin and hypercharge vector sources $v_{3}^{\mu}$ and $v_{8}^{\mu}$ as aids in computing the corresponding vector-current propagators. We shall require the chiral lagrangians $\mathcal{L}^{(2)}, \mathcal{L}^{(4)}$ and $\mathcal{L}^{(6)}$. These lagrangians will involve just the so-called normal vector currents. In this paper, we will not consider any of the anomalous currents. In the course of computing the one-loop and two-loop Feynman diagrams, we shall encounter divergent integrals to which we shall assign precise meaning via dimensional regularization. Due to the many terms which occur, the calculation is both difficult and complicated. Fortunately, it will be possible to check the correctness of our results along the way in a variety of ways. 


\section{One-loop and Two-loop Analyses}

We shall divide discussion of our calculation into three separate parts - loop analysis, counterterm analysis, and renormalization procedure. This section begins the process with a description of one-loop and two-loop amplitudes which are obtained by using the vector-source approach of Section 2. The Feynman diagrams underlying our loop and counterterm analyses are the ones given in Fig. (3) and Fig. (4). Throughout, we shall denote all contributions from the chiral $\mathcal{L}^{(4)}$ by a small circle enclosing a cross and those from $\mathcal{L}^{(6)}$ by a small square enclosing a cross. It turns out that all the Feynman integrals we shall encounter can ultimately be expressed in terms of just two quantities, which we call $A$ and $\bar{B}_{21}$. They are defined in Appendix A, where we also discuss in detail all such integrals occurring in our analysis. In our dimensional regularization treatment of these quantities, we have been very careful to allow throughout for sufficiently high powers in the smallness parameter $d-4$. As it happens, the only terms in our expansions which actually contribute are the ones we display in App. A.

\section{One-loop Amplitudes}

The one-loop chiral analysis of the isospin vector-current propagator was originally carried out by Gasser and Leutwyler in an $S U(2)$ basis of fields 1 . We shall briefly summarize the theoretical analysis with the $S U(3)$ basis employed in this paper and shall also include the one-loop expression for the hypercharge propagator. Our discussion of the one-loop sector will serve to set the stage for the two-loop analysis.

At one-loop level, the vector-current propagators are determined from the Feynman diagrams appearing in Fig. 3. There are two amplitudes, each arising from the chiral lagrangian $\mathcal{L}^{(2)}$, the unitarity diagram of Fig. 3(a) and the tadpole diagram of Fig. 3(b). For the isospin propagator, one must sum over contributions from both pion and kaon intermediate states whereas the hypercharge propagator gets contributions from just kaons.

It is the unitarity diagram which contains the 'real physics' such as the two-particle branch points. We shall explicitly consider the hypercharge case as it is somewhat less complicated. A straightforward calculation shows the invariant amplitude for the unitarity diagram of Fig. 3(a) to be

$$
\left.\mathcal{M}_{88}^{\mu \nu}\right|_{\text {unitarity }}=-3 i A\left(m_{K}^{2}\right) g^{\mu \nu}
$$




$$
-\left(q^{\mu} q^{\nu}-q^{2} g^{\mu \nu}\right)\left(\frac{i A\left(m_{K}^{2}\right)}{2 m_{K}^{2}}+6 i \bar{B}_{21}\left(q^{2}, m_{K}^{2}\right)\right) .
$$

The unitarity contribution cannot by itself be physical since it is noncovariant, divergent and scale-dependent. However, the tadpole amplitude

$$
\left.\mathcal{M}_{88}^{\mu \nu}\right|_{\operatorname{tad}}=3 i A\left(m_{K}^{2}\right) g^{\mu \nu}
$$

is seen to remove the problem of noncovariance in the unitarity amplitude via cancelation. The full hypercharge amplitude is then

$$
\left.\Pi_{88}\left(q^{2}\right)\right|_{1-\text { loop }}=-\frac{i A\left(m_{K}^{2}\right)}{2 m_{K}^{2}}-6 i \bar{B}_{21}\left(q^{2}, m_{K}^{2}\right)+\left.\Pi_{88}^{c t}\right|_{1-\text { loop }},
$$

where $\left.\Pi_{88}^{c t}\right|_{1-\text { loop }}$ contains the counterterms generated by the next-order chiral lagrangian $\mathcal{L}^{(4)}$.22 This latter contribution is shown in Fig. 3(c) and will be considered in Section 4.

The above discussion has concerned determination of just the hypercharge vector-current propagator at one-loop order. For completeness, we display without further discussion the one-loop amplitude for the isospin case $(a=b=3)$,

$$
\begin{aligned}
& \left.\Pi_{33}\left(q^{2}\right)\right|_{1-\text { loop }}=-\frac{i A\left(m_{\pi}^{2}\right)}{3 m_{\pi}^{2}}-\frac{i A\left(m_{K}^{2}\right)}{6 m_{K}^{2}} \\
& \quad-4 i \bar{B}_{21}\left(q^{2}, m_{\pi}^{2}\right)-2 i \bar{B}_{21}\left(q^{2}, m_{K}^{2}\right)+\left.\Pi_{33}^{c t}\right|_{1-\text { loop }} .
\end{aligned}
$$

A simple check of the calculation is obtained by passing to the $S U(3)$ limit of $m_{\pi}=m_{K}$. As expected, the isospin and hypercharge amplitudes agree.

\section{Two-loop Amplitudes}

In some sense, the content of this subsection must parallel that just given. One simply calculates the set of amplitudes associated with Feynman diagrams at two-loop order. However, in perturbative quantum field theory, things get worse as one proceeds to higher orders. Such is the case here. Instead of 2 types of non-counterterm amplitudes as in Fig. 3(a)-(b), there are now 9 non-counterterm amplitudes shown in Fig. 4(a)-(i). Of these, all but the 'eyeglass' diagram of Fig. 4(i) may be considered as corrections of one kind or another to the one-loop diagrams of Fig. 3. A detailed account 
describing evaluation of these diagrams is clearly too lengthy a process to reproduce here. However, we can perhaps convey to the reader some useful insights with the following observations:

1. Since the different $\pi, K$ and $\eta$ flavors can generally occur in each loop, there are many terms contributing to both the isospin and hypercharge two-loop amplitudes.

2. A number of diagrams have noncovariant $\left(g_{\mu \nu}\right)$ contributions but they all turn out to cancel, as they must.

3. Interestingly, some diagrams contain terms in which a divergent quantity is multiplied by a nonpolynomial function. Such terms are potentially disasterous to the calculational program since they cannot be removed by the usual renormalization procedure. However, they cancel totally.

4. Two-loop amplitudes contain a number of contributions from the chiral lagrangian $\mathcal{L}^{(4)}$, in addition to those from $\mathcal{L}^{(2)}$.

The final comment motivates introduction at this point of $\mathcal{L}^{(4)}$, the nextto-lowest order chiral lagrangian. 2] In principle, the entire $\mathcal{O}\left(q^{4}\right)$ lagrangian has three parts. The first has terms containing the meson field variable $U$,

$$
\begin{aligned}
\mathcal{L}^{(4)} & =\sum_{i=1}^{10} L_{i} O_{i} \\
& =L_{1}\left[\operatorname{Tr}\left(D_{\mu} U D^{\mu} U^{\dagger}\right)\right]^{2}+L_{2} \operatorname{Tr}\left(D_{\mu} U D_{\nu} U^{\dagger}\right) \cdot \operatorname{Tr}\left(D^{\mu} U D^{\nu} U^{\dagger}\right) \\
& +L_{3} \operatorname{Tr}\left(D_{\mu} U D^{\mu} U^{\dagger} D_{\nu} U D^{\nu} U^{\dagger}\right) \\
& +L_{4} \operatorname{Tr}\left(D_{\mu} U D^{\mu} U^{\dagger}\right) \operatorname{Tr}\left(\chi U^{\dagger}+U \chi^{\dagger}\right) \\
& +L_{5} \operatorname{Tr}\left(D_{\mu} U D^{\mu} U^{\dagger}\left(\chi U^{\dagger}+U \chi^{\dagger}\right)\right)+L_{6}\left[\operatorname{Tr}\left(\chi U^{\dagger}+U \chi^{\dagger}\right)\right]^{2} \\
& +L_{7}\left[\operatorname{Tr}\left(\chi^{\dagger} U-U \chi^{\dagger}\right)\right]^{2}+L_{8} \operatorname{Tr}\left(\chi U^{\dagger} \chi U^{\dagger}+U \chi^{\dagger} U \chi^{\dagger}\right) \\
& +i L_{9} \operatorname{Tr}\left(L_{\mu \nu} D^{\mu} U D^{\nu} U^{\dagger}+R_{\mu \nu} D^{\mu} U^{\dagger} D^{\nu} U\right) \\
& +L_{10} \operatorname{Tr}\left(L_{\mu \nu} U R^{\mu \nu} U^{\dagger}\right) .
\end{aligned}
$$

The second has additional contributions occurring at the same order but which contain only external sources,

$$
\mathcal{L}_{\text {ext }}=H_{1} \operatorname{Tr}\left(L_{\mu \nu} L^{\mu \nu}+R_{\mu \nu} R^{\mu \nu}\right)+H_{2} \operatorname{Tr}\left(\chi^{\dagger} \chi\right) .
$$


In the above, $L_{\mu \nu}, R_{\mu \nu}$ are the field-strength tensors

$$
\begin{aligned}
& L_{\mu \nu}=\partial_{\mu} \ell_{\nu}-\partial_{\nu} \ell_{\mu}+i\left[\ell_{\mu}, \ell_{\nu}\right] \\
& R_{\mu \nu}=\partial_{\mu} r_{\nu}-\partial_{\nu} r_{\mu}+i\left[r_{\mu}, r_{\nu}\right] .
\end{aligned}
$$

The third consists of two terms which vanish upon using the equations of motion,

$$
\mathcal{L}_{\text {eq }- \text { motn }}=G_{1} \operatorname{Tr}(\mathcal{E} \cdot \mathcal{E})+G_{2} \operatorname{Tr}\left(\mathcal{E} \cdot i\left(\chi^{\dagger} U-U^{\dagger} \chi\right)\right)
$$

where

$$
\mathcal{E} \equiv i\left(2 D_{\mu}\left(U^{\dagger} D^{\mu} U\right)+\chi^{\dagger} U-U^{\dagger} \chi-\frac{1}{3} \operatorname{Tr}\left(\chi^{\dagger} U-U^{\dagger} \chi\right) 1\right) .
$$

In applications up to one-loop order, these terms can be neglected since they contribute only through tree diagrams where the equations of motion $\mathcal{E}=0$ may be applied. This is no longer true for a two-loop calculation. The terms in $\mathcal{L}_{\text {eq-motn }}$ may in general contribute a polynomial to the amplitude, which in turn could be absorbed into the coupling constants of the $\mathcal{O}\left(q^{6}\right)$ counterterm lagrangian.11 In our application we have kept these terms at all stages of the calculation. Although present in intermediate steps, however, the effect of $G_{1}, G_{2}$ cancels completely in the final result.

For the hypercharge vector-current propagator the result of working out all the two-loop Feynman diagrams is

$$
\begin{aligned}
& \left.\Pi_{88}\left(q^{2}\right)\right|_{2-\mathrm{p}}=\frac{1}{F_{0}^{2}}\left[-24 q^{2} L_{9} i \bar{B}_{21}\left(q^{2}, m_{K}^{2}\right)+2 i A\left(m_{K}^{2}\right)\left[6 L_{10}+\left(6-\frac{q^{2}}{m_{K}^{2}}\right) L_{9}\right]\right. \\
& +q^{2}\left[\frac{1}{8}\left(\frac{i A\left(m_{K}^{2}\right)}{m_{K}^{2}}\right)^{2}+3 \frac{i A\left(m_{K}^{2}\right)}{m_{K}^{2}} i \bar{B}_{21}\left(q^{2}, m_{K}^{2}\right)+18\left(i \bar{B}_{21}\left(q^{2}, m_{K}^{2}\right)\right)^{2}\right] \\
& \quad-3 \frac{m_{K}^{2}}{q^{2}} i \bar{B}\left(q^{2}, m_{K}^{2}\right)\left[8\left(m_{\pi}^{2}+2 m_{K}^{2}\right)\left(L_{4}-2 L_{6}\right)+8 m_{K}^{2}\left(L_{5}-2 L_{8}\right)\right. \\
& \left.\left.-\frac{1}{3} i A\left(m_{\eta}^{2}\right)\right]\right]+\left.\Pi_{88}^{c t}\right|_{2-\text { loop }}
\end{aligned}
$$

where we display only implicitly the two-loop counterterm contribution

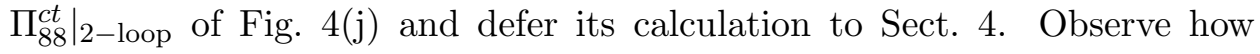
the above result reinforces the statement made earlier that all Feynman integrals in our analysis are reducible to just the two quantities $A$ and $\bar{B}_{21}$. 
To summarize, up to this point we have determined the isospin and hypercharge amplitudes through two-loop order,12

$$
\Pi_{a b}\left(q^{2}\right)=\left.\Pi_{a b}\left(q^{2}\right)\right|_{1-\text { loop }}+\left.\Pi_{a b}\left(q^{2}\right)\right|_{2-\text { loop }} \quad(a=b=3,8) .
$$

These results cannot yet be accepted as physically meaningful due to the presence of divergent terms and to the explicit dependence on an arbitrary scale $\mu$ which is an artifact of splitting loop and counterterm contributions.

\section{Enumeration of Counterterms}

The difficulties of divergence and scale-dependence in the 1-loop and 2-loop amplitudes are overcome by making appropriate use of counterterms. It is necessary to first generate a list at both 1-loop and 2-loop order of all counterterms which can possibly contribute. Carrying out this process for the $\mathcal{O}\left(q^{6}\right)$ counterterms will be the primary objective of this section.

Let us begin by reminding ourselves of which counterterms appear at $\mathcal{O}\left(q^{4}\right)$ order.2 2 Out of a total of 12 possible operators in the chiral lagrangians $\mathcal{L}^{(4)}$ of Eq. (23) and $\mathcal{L}_{\text {ext }}$ of Eq. (24), only the $L_{10}$ and $H_{1}$ terms turn out to have nonzero matrix elements taken between single external vector-source lines. One easily finds the $\mathcal{O}\left(q^{4}\right)$ counterterm amplitude to have the flavor-independent and covariant form,

$$
\left.\Pi_{a b}^{c t}\right|_{1-l o o p}=-2\left(L_{10}+2 H_{1}\right) \quad(a=b=3,8) .
$$

As noted earlier, the $\mathcal{O}\left(q^{4}\right)$ counterterm contribution appears in Fig. 3(c).

\section{Determination of the $\mathcal{O}\left(q^{6}\right)$ Counterterms}

Since the construction to follow will be somewhat formal in nature, it is important to keep in mind certain tenets of ChPT for guidance. Thus - we have found the two-loop calculations of $\Pi_{33}$ and $\Pi_{88}$ to produce ultraviolet divergences. The low energy counting scheme of ChPT implies that these divergencies can be absorbed into the coupling constants of counterterms of order $q^{6}$, thus renormalizing the amplitude. 13. However, more generally speaking all possible counterterms of order $q^{6}$ have to be included in order to obtain the most general amplitude consistent with chiral symmetry. This is irrespective of whether divergencies occur in the loop amplitude or not. 
In addition to those counterterms that contribute to $\Pi_{33}$ and $\Pi_{88}$, we shall also construct the set of $\mathcal{O}\left(q^{6}\right)$ counterterms which enter the two-loop analysis of the process $\gamma \gamma \rightarrow \pi^{0} \pi^{0}$ and $\eta \rightarrow \pi^{0} \gamma \gamma$. Our motivation stems in part from a recent analysis at two-loop order in ChPT of the first of these reactions. 119] In that work, however, no reference is made to a complete basis of $\mathcal{O}\left(q^{6}\right)$ counterterms. Our aim here will be to establish the precise relation between $\mathcal{O}\left(q^{6}\right)$ counterterms which contribute to both the $\gamma \gamma \rightarrow$ $\pi^{0} \pi^{0}$ amplitudes and the vector current two-point function. In addition, we hope that the details given below will serve to simplify comparison with future works in this subject.

Our first step is to derive a list of counterterms contributing a priori to the processes under consideration. Since we consider only couplings to neutral pseudoscalar mesons, the analysis simplifies considerably. There are no one-particle reducible diagrams involving $\mathcal{O}\left(q^{6}\right)$ counterterms. The reason is simply that to lowest order, $q^{2}$, the vector source couples to an even number of Goldstone bosons. We may therefore switch off the external fields except for the vector sources $v_{\mu}^{a}$. Furthermore, since all flavour matrices involved are diagonal, the situation can be described by

$$
\begin{aligned}
a_{\mu} & =0, \quad p=0, \quad s=\mathbf{m}, \\
\phi & =\text { diagonal } \rightarrow \quad U=\text { diagonal } .
\end{aligned}
$$

To proceed, we choose a set of building blocks transforming homogeneously under chiral transformations. The invariants can then be constructed easily by taking traces in flavor space. Our construction will closely follow the work in Refs. [14, 15]. The building blocks we use are

$$
\begin{aligned}
\chi_{ \pm} & =u\left(U^{\dagger} \chi \pm \chi^{\dagger} U\right) u^{\dagger}, \\
u_{\mu} & =i u^{\dagger} D_{\mu} U u^{\dagger}, \\
w_{\mu \nu} & =\nabla_{\mu} u_{\nu}+\nabla_{\nu} u_{\mu}, \\
f_{ \pm \mu \nu} & =u R_{\mu \nu} u^{\dagger} \pm u^{\dagger} L_{\mu \nu} u,
\end{aligned}
$$

as well as the totally antisymmetric tensor $\epsilon_{\mu \nu \rho \sigma}$. All the quantities $U, \chi$, $D_{\mu} U$ and the field strengths $L_{\mu \nu}, R_{\mu \nu}$ have been defined in previous sections and $u$ is the 'square-root' of the matrix-field $U$,

$$
U=u \cdot u
$$

\footnotetext{
${ }^{3}$ Throughout this article we work in chiral SU(3). Comparison to the constants appearing in Ref. 19] is therefore only possible after the $\mathrm{SU}(2)$ limit has been taken.
} 
Under a chiral $S U(3) \otimes S U(3)$ transformation, the matrix $u$ transforms as

$$
u(\phi) \rightarrow g_{R} u(\phi) h^{\dagger}(\phi)=h(\phi) u(\phi) g_{L}^{\dagger}
$$

where the nonlinear transformation $h(\phi)$ which first appears in Eq. (34) is, in fact, defined by this same equation. 116, 17 As a consequence, the left-chiral current $u_{\mu}$ transforms homogeneously,

$$
u_{\mu} \rightarrow h(\phi) u_{\mu}(\phi) h^{\dagger}(\phi)
$$

Given some object $\mathcal{O}$, a covariant derivative $\nabla_{\mu} \mathcal{O}$ which transforms in like manner to $u_{\mu}$ can be defined as

$$
\nabla_{\mu} \mathcal{O} \equiv \partial_{\mu} \mathcal{O}+\left[\Gamma_{\mu}, \mathcal{O}\right]
$$

where

$$
\Gamma_{\mu}=\frac{1}{2}\left(u^{\dagger}\left[\partial_{\mu}-i\left(v_{\mu}+a_{\mu}\right)\right] u+u\left[\partial_{\mu}-i\left(v_{\mu}-a_{\mu}\right)\right] u^{\dagger}\right)
$$

Here $v_{\mu}, a_{\mu}$ are the matrix-valued external fields with spin-one introduced in Sect. 2.

The building blocks of Eq. (32) as well as covariant derivatives thereof thus transform according to

$$
\mathcal{O} \rightarrow h(\phi) \mathcal{O} h^{\dagger}(\phi)
$$

The most general set of counterterms of order $q^{6}$ is then constructed by multiplying together the building blocks in Eq. (32) up to the appropriate order in the low energy expansion and then performing flavor traces like

$$
\operatorname{Tr}\left(\mathcal{O}_{i} \mathcal{O}_{j} \ldots\right), \quad \ldots, \quad \operatorname{Tr}\left(\mathcal{O}_{i} \mathcal{O}_{j} \ldots\right) \operatorname{Tr}\left(\mathcal{O}_{k} \ldots\right) \ldots \operatorname{Tr}\left(\mathcal{O}_{l} \ldots\right)
$$

In Eq. (39), Lorentz indices are to be contracted and symmetrizations performed such that the discrete symmetries $P$ and $C$ are conserved. The transformation properties of the various building blocks under $P$ and $C$ can be found in Ref. 114. We also note that all quantities in Eq. (32), except for $\chi_{ \pm}$, are traceless.

The procedure just described for constructing the invariants of order $q^{6}$ leads to a set of terms which is in general redundant. In principle this set of terms can be reduced further by the use of the equations of motion, trace and epsilon identities 18 as well as by partial integrations. We shall not 
elaborate further on such procedures, since the case we are interested in is much simpler and does not require that level of analysis.

Let us return to the restricted situation as characterized in Eq. (31). Under these circumstances the building blocks of Eq. (32) are transformed into

$$
\begin{array}{lll}
\chi_{ \pm}=2 B_{0} \mathbf{m}\left(U^{\dagger} \pm U\right), & f_{+\mu \nu}=2\left(\partial_{\mu} v_{\nu}-\partial_{\nu} v_{\mu}\right), \\
u_{\mu}=i u^{\dagger} \partial_{\mu} u^{\dagger}, & f_{-\mu \nu}=0 . \\
w_{\mu \nu}=\partial_{\mu} u_{\nu}+\partial_{\nu} u_{\mu}, & &
\end{array}
$$

The only nontrivial objects at our disposal are therefore $\chi_{+}, f_{+\mu \nu}$ and covariant derivatives thereof as well as the totally antisymmetric $\epsilon$-tensor. The covariant derivative is also simplified, and is explicitly given as

$$
\nabla_{\mu} \mathcal{O}=\partial_{\mu} \mathcal{O}-i\left[v_{\mu}, \mathcal{O}\right]
$$

Since only the diagonal isospin and hypercharge components $a=3,8$ are studied in this work, it follows that $\nabla_{\mu} \chi_{+} \sim \nabla_{\mu} M=0$. In the same manner, we see that $f_{+\mu \nu}$ must be linear in the vector source $v_{\mu}$, so two powers of $f_{+\mu \nu}$ are needed. As regards the operators $\chi_{-}$and $w_{\mu \nu}$, we note that $\chi_{-}$ involves only odd numbers of mesons and that $w_{\mu \nu}$ is parity-odd and hence cannot lead to an operator of the form we are looking for. Finally, the remaining two powers of 'small' momenta as well as the two pseudoscalar meson fields can only be provided by two powers of $u_{\mu}$ or one factor of $\chi_{+}$. We are thus left with an expression of the form

$$
\begin{aligned}
\mathcal{L}^{(6)}=\frac{1}{F_{0}^{2}} & \left(K_{1} \operatorname{Tr}\left(\nabla_{\lambda} f_{+}^{\mu \nu} \nabla^{\lambda} f_{+\mu \nu}\right)+K_{2} \operatorname{Tr}\left(\nabla_{\mu} f_{+}^{\mu \nu} \nabla^{\lambda} f_{+\lambda \nu}\right)\right. \\
& +K_{3} \operatorname{Tr}\left(f_{+\mu \nu} f_{+}^{\mu \nu} \chi_{+}\right)+K_{4} \operatorname{Tr}\left(f_{+\mu \nu} f_{+}^{\mu \nu}\right) \operatorname{Tr}\left(\chi_{+}\right) \\
& \left.+K_{10} \operatorname{Tr}\left(f_{+\mu \nu} f_{+}^{\mu \nu} u^{\rho} u_{\rho}\right)+K_{11} \operatorname{Tr}\left(f_{+\mu \nu} f_{+}^{\mu \rho} u^{\nu} u_{\rho}\right)+\ldots\right)
\end{aligned}
$$

The ellipses in this equation denote all possible independent operators which can arise from the terms containing $K_{10}$ and $K_{11}$ by changing the order of the building blocks $f_{+\mu \nu}$ and $u_{\rho}$ within the flavour trace and/or by taking two flavour traces. We shall refrain from writing down such contributions explicitly since they involve only diagonal flavour traces and thus lead to interactions of the type

$$
\begin{aligned}
\mathcal{L}_{\text {der }}^{(6)^{\prime}}= & \frac{4 e^{2}}{F_{0}^{4}} F_{\mu \nu} F^{\mu \rho}\left[\left(K_{10}^{\prime} g_{\sigma \tau} g^{\nu \rho}+K_{11}^{\prime} g_{\sigma}^{\nu} g_{\tau}^{\rho}\right) \operatorname{Tr}\left(Q^{2} \partial^{\sigma} \phi \partial^{\tau} \phi\right)\right. \\
& +\left(K_{12}^{\prime} g_{\sigma \tau} g^{\nu \rho}+K_{13}^{\prime} g_{\sigma}^{\nu} g_{\tau}^{\rho}\right) \operatorname{Tr}\left(Q^{2}\right) \operatorname{Tr}\left(\partial^{\sigma} \phi \partial^{\tau} \phi\right) \\
& \left.+\left(K_{14}^{\prime} g_{\sigma \tau} g^{\nu \rho}+K_{15}^{\prime} g_{\sigma}^{\nu} g_{\tau}^{\rho}\right) \operatorname{Tr}\left(Q \partial^{\sigma} \phi\right) \operatorname{Tr}\left(Q \partial^{\tau} \phi\right)\right] .
\end{aligned}
$$


In the above, we define the electromagnetic field strength

$$
F_{\mu \nu} \equiv \partial_{\mu} A_{\nu}-\partial_{\nu} A_{\mu}
$$

where $A_{\mu}$ is the photon field, and take

$$
v_{\mu}=e Q A_{\mu}, \quad Q=\frac{1}{3} \operatorname{diag}(2,-1,-1) .
$$

It is now straightforward to determine the counterterm contributions to $\Pi_{33}$ and $\Pi_{88}$ which arise from the lagrangian $\mathcal{L}^{(6)}$. We obtain

$$
\left.\Pi_{33}^{c t}\left(q^{2}\right)\right|_{2-\text { loop }}=-\frac{4}{F_{0}^{2}}\left[q^{2}\left(2 K_{1}+K_{2}\right)+4 m_{\pi}^{2} K_{3}+4\left(2 m_{K}^{2}+m_{\pi}^{2}\right) K_{4}\right]
$$

and

$$
\left.\Pi_{88}^{c t}\left(q^{2}\right)\right|_{2-\text { loop }}=-\frac{4}{F_{0}^{2}}\left[q^{2}\left(2 K_{1}+K_{2}\right)+4 m_{\eta}^{2} K_{3}+4\left(2 m_{K}^{2}+m_{\pi}^{2}\right) K_{4}\right],
$$

where the lowest order expressions for squares of the meson masses ( $c f$. Eq. (10) ) have been employed. Note that the contribution of $q^{6}$ counterterms to the difference, $\Pi_{33}-\Pi_{88}$, is a constant depending only on $K_{3}$. Also, from the four terms $K_{1}, \ldots K_{4}$ which may contribute a priori, only three independent combinations actually appear. The renormalization of coupling constants such as $K_{1}, \ldots K_{4}$ as well as their properties following from the renormalization group equations will be discussed in Sect. 5 .

As for the two-meson two-photon processes, we first note a relation between $\gamma \gamma \rightarrow \pi^{0} \pi^{0}$ and $\eta \rightarrow \pi^{0} \gamma \gamma$. Upon evaluating the flavour traces in Eqs. (42), (44) we easily see that as long as the pieces which violate the Zweig rule are neglected (i.e. those terms with two flavour traces), then the amplitudes for $\gamma \gamma \rightarrow \pi^{0} \pi^{0}$ and $\eta \rightarrow \pi^{0} \gamma \gamma$ will depend on the same linear combination of the $\left\{K_{i}\right\}$. 21] Let us not pursue this line of reasoning any further, but instead concentrate in the following on the $\gamma \gamma \rightarrow \pi^{0} \pi^{0}$ reaction. The $\gamma \gamma \rightarrow \pi^{0} \pi^{0}$ matrix element can be written as

$$
{ }_{\text {out }}\left\langle\pi^{0}\left(p_{1}\right) \pi^{0}\left(p_{2}\right) \mid \gamma\left(q_{1}, \epsilon_{1}\right) \gamma\left(q_{2}, \epsilon_{2}\right)\right\rangle_{\text {in }}=i(2 \pi)^{4} \delta^{(4)}\left(P_{f}-P_{i}\right) e^{2} \epsilon_{1}^{\mu} \epsilon_{2}^{\nu} V_{\mu \nu}
$$

with

$$
V^{\mu \nu}=A(s, t, u) T_{1}^{\mu \nu}+B(s, t, u) T_{2}^{\mu \nu} .
$$

The tensors $T_{1,2}^{\mu \nu}$ are defined in Ref. [19], and we shall adopt the notation and conventions used there. It is straightforward to determine the contribution 
of $\mathcal{O}\left(q^{6}\right)$ counterterms to the Lorentz invariant amplitudes $A, B$, and we find

$$
\begin{aligned}
& A^{\mathrm{ct}}(s, t, u)=\frac{4}{F_{0}^{4}}\left[-8 M_{\pi}^{2}\left(-\frac{5}{9} K_{3}-\frac{4}{3} K_{4}+\frac{5}{9} K_{10}^{\prime}+\frac{4}{3} K_{12}^{\prime}+K_{14}^{\prime}\right)\right. \\
& \left.+s\left(\frac{5}{18}\left(8 K_{10}^{\prime}+K_{11}^{\prime}\right)+\frac{2}{3}\left(8 K_{12}^{\prime}+K_{13}^{\prime}\right)+4 K_{14}^{\prime}+\frac{1}{2} K_{15}^{\prime}\right)\right] \\
& B^{\mathrm{ct}}(s, t, u)=-\frac{1}{F_{0}^{4}}\left[\frac{5}{9} K_{11}^{\prime}+\frac{4}{3} K_{13}^{\prime}+K_{15}^{\prime}\right] .
\end{aligned}
$$

This completes our discussion of the counterterm contribution to the $\gamma \gamma \rightarrow$ $\pi^{0} \pi^{0}$ reaction. Observe that the derivative lagrangian also contributes a constant piece to the amplitude $A$. The information contained in the twoloop expression for the vector propagator is thus not sufficient to fix the constant shift in A at this order of the low energy expansion.

\section{Renormalization Prescription}

Now that we have a complete set of $\mathcal{O}\left(q^{4}\right)$ and $\mathcal{O}\left(q^{6}\right)$ counterterms, the next step is to formulate a procedure which allows the divergences to be subtracted and the scale-dependence removed from the calculation.

\section{Subtraction Procedure}

There exist different ways of implementing these steps, e.g. minimal subtraction, etc. We have found it most convenient to subtract in the quantity $\bar{\lambda}$. This amounts to expanding the various counterterms in decreasing

powers of $\bar{\lambda}$. In the following, let $K$ denote any of the $\mathcal{O}\left(q^{6}\right)$ counterterms and $L$ represent any of the $\mathcal{O}\left(q^{4}\right)$ counterterms. For the former, we write

$$
\begin{aligned}
K & =\mu^{2(d-4)} \sum_{n=2}^{-\infty} K^{(n)}(\mu) \bar{\lambda}^{n} \\
& =\mu^{2(d-4)}\left[K^{(2)}(\mu) \bar{\lambda}^{2}+K^{(1)}(\mu) \bar{\lambda}+K^{(0)}(\mu)+\ldots\right],
\end{aligned}
$$

whereas the latter is given by

$$
\begin{aligned}
L & =\mu^{d-4} \sum_{n=1}^{-\infty} L^{(n)}(\mu) \bar{\lambda}^{n} \\
& =\mu^{d-4}\left[L^{(1)}(\mu) \bar{\lambda}+L^{(0)}(\mu)+L^{(-1)}(\mu) \bar{\lambda}^{-1}+\ldots\right] .
\end{aligned}
$$


In our two-loop analysis, we shall actually need only the $K^{(2)}, K^{(1)}, K^{(0)}$ and the $L^{(1)}, L^{(0)}, L^{(-1)}$ counterterms.

The definition of $\mathcal{O}\left(q^{4}\right)$ counterterms used here has a simple association with that originally introduced by Gasser and Leutwyler in their papers on one-loop chiral perturbation theory. We restrict our attention to Ref. [2] since, like this paper, it too employs an $S U(3)$ basis. Then starting from Eq. (7.25) of Ref. [2],

$$
\begin{aligned}
L_{i}^{(\mathrm{G}-\mathrm{L})} & =\Gamma_{i} \lambda+L_{i}^{r} \quad(i=1, \ldots, 10) \\
H_{i}^{(\mathrm{G}-\mathrm{L})} & =\Delta_{i} \lambda+H_{i}^{r} \quad(i=1,2)
\end{aligned}
$$

we see that our $\left\{L_{i}^{(n)}\right\}$ and $\left\{H_{i}^{(n)}\right\}$ are related to the Gasser-Leutwyler parameters by

$$
\begin{aligned}
& L_{i}^{(1)}=\Gamma_{i} \quad \text { and } \quad L_{i}^{(0)}=L_{i}^{r} \quad(i=1, \ldots, 10) \\
& H_{i}^{(1)}=\Delta_{i} \quad \text { and } \quad H_{i}^{(0)}=H_{i}^{r} \quad(i=1,2) \text {. }
\end{aligned}
$$

\section{Scale Dependence}

Having chosen a subtraction procedure, we must next determine the scale dependence of the counterterm coefficients. This is done by first noting that although the full counterterm is independent of scale,

$$
\mu \frac{d K}{d \mu}=0 \quad \text { and } \quad \mu \frac{d L}{d \mu}=0
$$

the $\left\{K^{(n)}\right\}$ and $\left\{L^{(n)}\right\}$ must themselves generally be scale-dependent in order to compensate the scale-dependence of the prefactors, $\mu^{2(d-4)}$ in Eq. (51) and $\mu^{d-4}$ in Eq. (52). Insertion of Eq. (51) into the first equation of Eq. (55) yields an infinite sequence of differential relations for the $\mathcal{O}\left(q^{6}\right)$ counterterms,

$$
\begin{aligned}
& \mu \frac{d K^{(2)}}{d \mu}=0 \\
& \mu \frac{d K^{(1)}}{d \mu}=-\frac{2}{16 \pi^{2}} K^{(2)} \\
& \mu \frac{d K^{(0)}}{d \mu}=\frac{2}{16 \pi^{2}}\left[\frac{C}{16 \pi^{2}} K^{(2)}-K^{(1)}\right] .
\end{aligned}
$$


where, in terms of the Euler constant $\gamma$, we define

$$
C \equiv \frac{1}{2}(\ln (4 \pi)-\gamma+1)
$$

An analogous but distinct set of relations is obtained for the $\mathcal{O}\left(q^{4}\right)$ counterterms by inserting Eq. (52) into the second equation of Eq. (55),

$$
\begin{aligned}
\mu \frac{d L^{(1)}}{d \mu} & =0 \\
\mu \frac{d L^{(0)}}{d \mu} & =-\frac{1}{16 \pi^{2}} L^{(1)} \\
\mu \frac{d L^{(-1)}}{d \mu} & =\frac{1}{16 \pi^{2}}\left[\frac{C}{16 \pi^{2}} L^{(1)}-L^{(0)}\right] .
\end{aligned}
$$

It is straightforward to sequentially solve relations of the type given above, and we find for the first few of the $\left\{K^{(n)}\right\}$,

$$
\begin{aligned}
K^{(2)}(\mu)= & \left.K^{(2)}\left(\mu_{0}\right) \quad \text { (independent of } \mu\right) \\
K^{(1)}(\mu)= & K^{(1)}\left(\mu_{0}\right)+\frac{K^{(2)}}{16 \pi^{2}} \ln \left(\frac{\mu_{0}^{2}}{\mu^{2}}\right) \\
K^{(0)}(\mu)= & K^{(0)}\left(\mu_{0}\right)+\frac{1}{16 \pi^{2}}\left[\left(K^{(1)}\left(\mu_{0}\right)-\frac{C K^{(2)}}{16 \pi^{2}}\right) \ln \left(\frac{\mu_{0}^{2}}{\mu^{2}}\right)\right. \\
& \left.+\frac{K^{(2)}}{32 \pi^{2}} \ln ^{2}\left(\frac{\mu_{0}^{2}}{\mu^{2}}\right)\right],
\end{aligned}
$$

whereas the sequence of $\left\{L^{(n)}\right\}$ begins as

$$
\begin{aligned}
L^{(1)}(\mu)= & \left.L^{(1)}\left(\mu_{0}\right) \quad \text { (independent of } \mu\right) \\
L^{(0)}(\mu)= & L^{(0)}\left(\mu_{0}\right)+\frac{L^{(1)}}{32 \pi^{2}} \ln \left(\frac{\mu_{0}^{2}}{\mu^{2}}\right) \\
L^{(-1)}(\mu)= & L^{(-1)}\left(\mu_{0}\right)+\frac{1}{32 \pi^{2}}\left[\left(L^{(0)}\left(\mu_{0}\right)-\frac{C L^{(1)}}{16 \pi^{2}}\right) \ln \left(\frac{\mu_{0}^{2}}{\mu^{2}}\right)\right. \\
& \left.+\frac{L^{(1)}}{64 \pi^{2}} \ln ^{2}\left(\frac{\mu_{0}^{2}}{\mu^{2}}\right)\right] .
\end{aligned}
$$


Up to this point, the $\left\{K^{(n)}\right\}$ and $\left\{L^{(n)}\right\}$ are arbitrary. In Sect. 6, we shall show how to constrain the counterterms which are relevant to our calculation by imposing conditions of finiteness and scale-independence.

\section{Effect of Mass Renormalization}

For the final topic in this Section, we consider renormalization of the meson mass parameters. This intermediate step turns out to considerably simplify the form of both the isospin and hypercharge vector-current propagators $\Pi_{33}$ and $\Pi_{88}$. For definiteness, we shall again explicitly study just $\Pi_{88}$ and thus restrict our attention to the renormalization of the kaon mass parameter.

Recall in Ref. [2] that for kaons, the relation between the bare mass parameter $m_{K}$ and the radiatively-corrected quantity $M_{K}$ was shown to be

$$
m_{K}^{2}=M_{K}^{2}+\Delta_{K}
$$

where

$$
\begin{aligned}
\Delta_{K}= & \frac{1}{F_{0}^{2}}\left[8 M_{K}^{2}\left(M_{\pi}^{2}+2 M_{K}^{2}\right)\left(L_{4}^{(0)}-2 L_{6}^{(0)}\right)\right. \\
& \left.+8 M_{K}^{4}\left(L_{5}^{(0)}-2 L_{8}^{(0)}\right)-\frac{M_{K}^{2} M_{\eta}^{2}}{48 \pi^{2}} \ln \left(\frac{M_{\eta}^{2}}{\mu^{2}}\right)\right] .
\end{aligned}
$$

For the two-loop amplitude, one can replace $m_{K}^{2}$ by $M_{K}^{2}$ everywhere since the error made occurs in still higher order. However, at one-loop order Eqs.61),(62) must be utilized everywhere, i.e. both where $m_{K}^{2}$ appears explicitly and also where it occurs implicitly within a function. The effect of this substitution is

$$
\left.\Pi_{88}\left(q^{2}, m_{K}^{2}\right)\right|_{1-\text { loop }}=\left.\Pi_{88}\left(q^{2}, M_{K}^{2}\right)\right|_{1-\text { loop }}+\frac{3 \Delta_{K}}{q^{2}} i \bar{B}\left(q^{2}, M_{K}^{2}\right) .
$$

It can be shown that the additive term in Eq. (63) has the effect of cancelling the final term in the form for $\left.\Pi_{88}\left(q^{2}\right)\right|_{2-\text { loop }}$ appearing in Eq. (28). This leaves us with the more compact expression,

$$
\begin{aligned}
& \left.\Pi_{88}\left(q^{2}, M_{K}^{2}\right)\right|_{2-\text { loop }}=\frac{1}{F_{0}^{2}}\left[-24 L_{9} q^{2} i \bar{B}_{21}\left(q^{2}, M_{K}^{2}\right)\right. \\
& +2 i A\left(M_{K}^{2}\right)\left[6 L_{10}+\left(6-\frac{q^{2}}{M_{K}^{2}}\right) L_{9}\right]+q^{2}\left[\frac{1}{8}\left(\frac{i A\left(M_{K}^{2}\right)}{M_{K}^{2}}\right)^{2}\right. \\
& \left.\left.+3 \frac{i A\left(M_{K}^{2}\right)}{M_{K}^{2}} i \bar{B}_{21}\left(q^{2}, M_{K}^{2}\right)+18\left(i \bar{B}_{21}\left(q^{2}, M_{K}^{2}\right)\right)^{2}\right]\right]
\end{aligned}
$$


The isospin amplitude behaves analogously, except now both pion and kaon mass renormalizations must be administered.

\section{Results}

We now have sufficient theoretical machinery to obtain our final representations for the isospin and hypercharge vector-current propagators. The first step in this process is to expand the one-loop and two-loop expressions obtained earlier in powers of the singular quantity $\bar{\lambda}$. When combined with the counterterm contributions which were derived in the previous section, the full amplitude is rendered finite and free of the arbitrary scale $\mu$ by appropriate choices of the renormalization constants contained within the counterterms. The discussion becomes clearest if we first consider the oneloop case in some detail, and then touch on the high points of the more complicated two-loop sector.

\section{Renormalization at One-loop Order}

It is again convenient to refer to the simpler hypercharge one-loop amplitude to illustrate the process. From Eq. (21) of Sect. 3 and Eq. (92) of App. A, we have

$$
\begin{aligned}
\left.\Pi_{88}\left(q^{2}\right)\right|_{1-\text { loop }}= & -2 L_{10}-4 H_{1}-\frac{i A\left(M_{K}^{2}\right)}{2 M_{K}^{2}}-6 i \bar{B}_{21}\left(q^{2}, M_{K}^{2}\right) \\
= & -\mu^{d-4}\left[\left(1+2 L_{10}^{(1)}+4 H_{1}^{(1)}\right) \cdot \bar{\lambda}\right. \\
& \left.+2 L_{10}^{(0)}\left(M_{K}^{2}\right)+4 H_{1}^{(0)}\left(M_{K}^{2}\right)\right]-6 i \bar{B}_{21}\left(q^{2}, M_{K}^{2}\right)
\end{aligned}
$$

Note that we have adopted the convenient renormalization scale of $M_{K}^{2}$ for the counterterms $L_{10}^{(0)}$ and $H_{1}^{(0)}$. Of course, any other choice is also allowed, but there will be compensating logarithmic factors as in Eq. (60).

The next step is the crucial one. One removes simultaneously the divergent factor of $\bar{\lambda}$ and dependence on the scale $\mu$ with the choice

$$
2 L_{10}^{(1)}+4 H_{1}^{(1)}=-1 .
$$

This result is consistent with that Ref. [2], where background field renormalization yielded the individual values $L_{10}^{(1)}=-1 / 4$ and $H_{1}^{(1)}=-1 / 8$. We 
are then left with the finite and scale-independent one-loop result

$$
\left.\Pi_{88}\left(q^{2}\right)\right|_{1-\text { loop }}=-2 L_{10}^{(0)}\left(M_{K}^{2}\right)-4 H_{1}^{(0)}\left(M_{K}^{2}\right)-6 i \bar{B}_{21}\left(q^{2}, M_{K}^{2}\right) .
$$

\section{Renormalization at Two-loop Order}

Renormalization at two-loop order proceeds in like manner, but with many more terms. Since the underlying logic is the same, we shall not dwell on the many details of the analysis. However, it is of some interest to observe that the leading degree of divergence at two-loops is quadratic,

$$
\begin{gathered}
\left.\Pi_{88}\left(q^{2}\right)\right|_{2-\text { loop }}=-\mu^{2(4-d)} \bar{\lambda}^{2}\left(\frac{4 q^{2}}{F_{0}^{2}}\left[\frac{1}{8}+2 K_{1}^{(2)}+K_{2}^{(2)}\right]\right. \\
\left.+\frac{32 M_{K}^{2}}{F_{0}^{2}}\left[K_{4}^{(2)}+\frac{2}{3} K_{3}^{(2)}\right]+\frac{16 M_{\pi}^{2}}{F_{0}^{2}}\left[K_{4}^{(2)}-\frac{1}{3} K_{3}^{(2)}\right]\right)+\ldots,
\end{gathered}
$$

where we have exhibited only the most singular terms. The $\bar{\lambda}^{2}$ divergences are removed by means of the scale-independent choices

$$
2 K_{1}^{(2)}+K_{2}^{(2)}=-\frac{1}{8} \quad \text { and } \quad K_{3}^{(2)}=K_{4}^{(2)}=0 .
$$

Analogous procedures for the remaining part of the amplitude turn out not to constrain the $\mathcal{O}\left(q^{6}\right)$ counterterms in terms of numerical values as in the above equation. Rather, relations are obtained which connect the $\mathcal{O}\left(q^{6}\right)$ counterterms with those from the $\mathcal{O}\left(q^{4}\right)$ sector,

$$
\begin{aligned}
2 K_{1}^{(1)}(\mu)+K_{2}^{(1)}(\mu) & =-L_{9}^{(0)}(\mu), \\
K_{3}^{(1)}(\mu) & =\frac{3}{4}\left[L_{9}^{(0)}(\mu)+L_{10}^{(0)}(\mu)\right], \\
K_{4}^{(1)}(\mu) & =\frac{1}{4}\left[L_{9}^{(0)}(\mu)+L_{10}^{(0)}(\mu)\right] .
\end{aligned}
$$

This completes the list of constraints.

\section{Final Form of Vector-current Propagators}

We express our final result for $\Pi_{88}$ in terms of the function $i \bar{B}_{21}$ and various counterterms, all fixed at scale $M_{K}^{2}$,

$$
\Pi_{88}\left(q^{2}, M_{K}^{2}\right)=
$$




$$
\begin{aligned}
& -2 L_{10}^{(0)}\left(M_{K}^{2}\right)-4 H_{1}^{(0)}\left(M_{K}^{2}\right)-6 i \bar{B}_{21}\left(q^{2}, M_{K}^{2}\right) \\
& +\frac{q^{2}}{F_{0}^{2}}\left[18\left(i \bar{B}_{21}\left(q^{2}, M_{K}^{2}\right)\right)^{2}-24 L_{9}^{(0)}\left(M_{K}^{2}\right) i \bar{B}_{21}\left(q^{2}, M_{K}^{2}\right)-P\left(M_{K}^{2}\right)\right] \\
& -\frac{4 M_{\pi}^{2}}{F_{0}^{2}}\left[R\left(M_{K}^{2}\right)-\frac{1}{3} Q\left(M_{K}^{2}\right)\right]-\frac{8 M_{K}^{2}}{F_{0}^{2}}\left[R\left(M_{K}^{2}\right)+\frac{2}{3} Q\left(M_{K}^{2}\right)\right],
\end{aligned}
$$

where we define

$$
\begin{aligned}
P(\mu) & \equiv 4\left[2 K_{1}^{(0)}(\mu)+K_{2}^{(0)}(\mu)+L_{9}^{(-1)}(\mu)\right], \\
Q(\mu) & \equiv 4 K_{3}^{(0)}(\mu)-3\left[L_{9}^{(-1)}(\mu)+L_{10}^{(-1)}(\mu)\right], \\
R(\mu) & \equiv 4 K_{4}^{(0)}(\mu)-L_{9}^{(-1)}(\mu)-L_{10}^{(-1)}(\mu) .
\end{aligned}
$$

The quantities $P, Q, R$ are the three new independent counterterms introduced at two-loop order. Observe that $L_{9}^{-1}$ and $L_{10}^{-1}$ have been absorbed in these quantities. This is an example of a general property of the generating functional, as pointed out in Ref. [19].

We can characterize the content of $\Pi_{88}$ as given above in terms of 'chiral counting'. Thus the first line on the right hand side is the one-loop result, whereas the remaining lines contain higher-order (two-loop) contributions which are proportional to the perturbative parameters of smallness $q^{2} / F_{0}^{2}$, $M_{\pi}^{2} / F_{0}^{2}, M_{K}^{2} / F_{0}^{2}$. For completeness, we also write down the analogous but more cumbersome result for $\Pi_{33}$,

$$
\begin{aligned}
& \Pi_{33}\left(q^{2}, M_{\pi}^{2}, M_{K}^{2}\right)=\frac{1}{48 \pi^{2}} \ln \frac{M_{K}^{2}}{M_{\pi}^{2}}-\frac{8 M_{K}^{2}}{F_{0}^{2}} R\left(M_{K}^{2}\right) \\
& -2 L_{10}^{(0)}\left(M_{K}^{2}\right)-4 H_{1}^{(0)}\left(M_{K}^{2}\right)-4 i \bar{B}_{21}\left(q^{2}, M_{\pi}^{2}\right)-2 i \bar{B}_{21}\left(q^{2}, M_{K}^{2}\right) \\
& +\frac{q^{2}}{F_{0}^{2}}\left[8\left(i \bar{B}_{21}\left(q^{2}, M_{\pi}^{2}\right)\right)^{2}+2\left(i \bar{B}_{21}\left(q^{2}, M_{K}^{2}\right)\right)^{2}+\frac{1}{18}\left(\frac{1}{16 \pi^{2}} \ln \frac{M_{K}^{2}}{M_{\pi}^{2}}\right)^{2}\right. \\
& +8 i \bar{B}_{21}\left(q^{2}, M_{\pi}^{2}\right) i \bar{B}_{21}\left(q^{2}, M_{K}^{2}\right)-P\left(M_{K}^{2}\right)+\frac{L_{9}^{(0)}\left(M_{K}^{2}\right)}{12 \pi^{2}} \ln \frac{M_{K}^{2}}{M_{\pi}^{2}} \\
& \left.-\left(2 i \bar{B}_{21}\left(q^{2}, M_{\pi}^{2}\right)+i \bar{B}_{21}\left(q^{2}, M_{K}^{2}\right)\right) \cdot\left(8 L_{9}^{(0)}\left(M_{K}^{2}\right)+\frac{1}{24 \pi^{2}} \ln \frac{M_{K}^{2}}{M_{\pi}^{2}}\right)\right] \\
& -\frac{4 M_{\pi}^{2}}{F_{0}^{2}}\left[Q\left(M_{K}^{2}\right)+R\left(M_{K}^{2}\right)+\frac{L_{9}^{(0)}\left(M_{K}^{2}\right)+L_{10}^{(0)}\left(M_{K}^{2}\right)}{8 \pi^{2}} \ln \frac{M_{K}^{2}}{M_{\pi}^{2}}\right] .
\end{aligned}
$$

After some algebra, one finds that the expressions in Eq. (71) and Eq. (73) agree in the $S U(3)$ limit. 


\section{Applications}

The vector current two-point functions may be expressed in terms of the corresponding Källén-Lehman spectral functions. Combined with the high energy behaviour which follows from perturbative QCD, the low energy representations of the two-point functions derived in the previous section is seen to contain two pieces of information: i) it yields the leading and nextto-leading order contributions to the low energy expansion of the spectral functions, and ii) it implies a set of sum rules which the spectral functions must satisfy. We shall discuss in turn the consequences of our results for i) and ii).

\section{Vector Spectral Functions}

An important application of our results involves determining the spectral functions associated with the isospin and hypercharge vector currents. As pointed out in the Introduction, the former is of special interest since it can be compared directly with existing data. Thus it is the isospin spectral function $\rho_{\mathrm{V} 33}\left(q^{2}\right)$ that will be discussed here.

From Eqs. (6)-(8), it follows that the relation between $\Pi_{33}\left(q^{2}\right)$ and $\rho_{\mathrm{V} 33}\left(q^{2}\right)$ is simply

$$
\Pi_{33}\left(q^{2}\right)=\int_{s_{0}}^{\infty} d s \frac{\rho_{\mathrm{V} 33}(s)}{s-q^{2}-i \epsilon}
$$

where $s_{0}=4 M_{\pi}^{2}$ is the threshold value. Thus the spectral function $\rho_{\mathrm{V} 33}\left(q^{2}\right)$ is proportional to the imaginary part of the propagator,

$$
\operatorname{Im} \Pi_{33}\left(q^{2}\right)=\pi \theta\left(q^{2}-4 m_{\pi}^{2}\right) \rho_{\mathrm{V} 33}\left(q^{2}\right) .
$$

A modest amount of effort in taking the imaginary part of Eq. (73) then yields the expression

$$
\begin{aligned}
& \rho_{\mathrm{V} 33}\left(q^{2}\right)=\frac{1}{48 \pi^{2}}\left(1-\frac{4 M_{\pi}^{2}}{q^{2}}\right)^{3 / 2} \theta\left(q^{2}-4 M_{\pi}^{2}\right) \\
& \times\left[1+\frac{q^{2}}{2 F_{0}^{2}}\left(8 L_{9}^{(0)}\left(M_{K}^{2}\right)+\frac{1}{24 \pi^{2}} \ln \frac{M_{K}^{2}}{M_{\pi}^{2}}-8 \operatorname{Re} i \bar{B}_{21}\left(q^{2}, M_{\pi}^{2}\right)\right.\right. \\
& \left.\left.\quad-4 \operatorname{Re} i \bar{B}_{21}\left(q^{2}, M_{K}^{2}\right)\right)\right]+\frac{1}{96 \pi^{2}}\left(1-\frac{4 M_{K}^{2}}{q^{2}}\right)^{3 / 2} \theta\left(q^{2}-4 M_{K}^{2}\right)
\end{aligned}
$$




$$
\begin{aligned}
& \times\left[1+\frac{q^{2}}{2 F_{0}^{2}}\left(8 L_{9}^{(0)}\left(M_{K}^{2}\right)+\frac{1}{24 \pi^{2}} \ln \frac{M_{K}^{2}}{M_{\pi}^{2}}\right.\right. \\
& \left.\left.-8 \operatorname{Re} i \bar{B}_{21}\left(q^{2}, M_{\pi}^{2}\right)-4 \operatorname{Re} i \bar{B}_{21}\left(q^{2}, M_{K}^{2}\right)+\right)\right] .
\end{aligned}
$$

This result can be directly compared with data. Since such comparison is performed near the $\pi \pi$ threshold, only the part of the above formula proportional to $\theta\left(q^{2}-4 M_{\pi}^{2}\right)$ contributes. Some care must be exercised in evaluating Re $i \bar{B}_{21}\left(q^{2}, M_{\pi}^{2}\right)$ and especially $\operatorname{Re} i \bar{B}_{21}\left(q^{2}, M_{K}^{2}\right)$.

The one-loop result was already depicted in Fig. 1. A more complete comparison appears in Fig. 5, where we display the individual one-loop, two-loop, and total chiral predictions together with spectral function data. The two-loop result given in Eq. (76) contains several additive contributions. Of these, the largest arises from the one containing the one-loop counterterm $L_{9}^{(0)}\left(M_{K}^{2}\right)$. For our numerical work, we have adopted the value of $L_{9}^{(0)}\left(M_{\eta}^{2}\right)$ cited in Ref. [9], with a small correction for working at scale $M_{K}^{2}$,

$$
\begin{aligned}
L_{9}^{(0)}\left(M_{K}^{2}\right) & =L_{9}^{(0)}\left(M_{\eta}^{2}\right)+\frac{1}{128 \pi^{2}} \log \left(\frac{M_{\eta}^{2}}{M_{K}^{2}}\right), \\
& =0.0071 \pm 0.0003+0.0002=0.0073 \pm 0.0003 .
\end{aligned}
$$

Another issue associated with a numerical analysis of $\rho_{\mathrm{V} 33}\left(q^{2}\right)$ is the value to be taken for the bare decay constant $F_{0}(c f$ Eq. (76)). We have followed standard procedure by employing the physical value

$$
F_{0} \quad \rightarrow \quad F_{\pi} \simeq 0.093 \mathrm{GeV},
$$

since any difference between $F_{0}$ and $F_{\pi}$ will be relegated to still higher order. The effect of using the one-loop result $2 F_{0}<F_{\pi}$ would be to increase the two-loop contribution relative to that shown in Fig. 5. Finally, we caution that some care be taken in interpreting a graph such as the one in Fig. 5. For the purpose of illustration, we have depicted the energy-dependence up to the value $s=12 m_{\pi}^{2}$. However, before that value is reached one expects that still higher-loop effects should be taken into account. In addition, due to its stronger $s$ dependence, the two-loop 'perturbation' ultimately becomes as large as the 'dominant' one-loop amplitude. From Fig. 5, it would appear that our calculation is valid up to $s \simeq 8 m_{\pi}^{2}$ or an energy of about $400 \mathrm{MeV}$.

\section{Chiral sum rules}


The real part of the vector current propagator depends on low energy constants appearing at $\mathcal{O}\left(q^{6}\right)$ in the chiral expansion. Some of these constants can be related to data by exploiting the high energy behaviour of QCD. We are thus able to derive a set of new chiral sum rules.

The asymptotic behavior of the functions $\Pi_{a a}\left(q^{2}\right)(a=3,8$ not summed $)$ is known from the operator product expansion, 22, 23.

$$
\Pi_{a a}\left(q^{2}\right) \sim \frac{1}{8 \pi^{2}}\left(1+\frac{\alpha_{s}\left(q^{2}\right)}{\pi}\right) \ln \frac{\mu^{2}}{-q^{2}} \quad(a=3,8),
$$

where $\mu$ is the renormalization scale and $\alpha_{s}\left(q^{2}\right)$ is the QCD running coupling constant. It follows from the asymptotic form that the difference $\Pi_{33}-\Pi_{88}$ satisfies the unsubtracted dispersion relation,

$$
\left(\Pi_{33}-\Pi_{88}\right)\left(q^{2}\right)=\int_{s_{0}}^{\infty} d s \frac{\left(\rho_{\mathrm{V} 33}-\rho_{\mathrm{V} 88}\right)(s)}{s-q^{2}-i \epsilon} .
$$

The real part of this dispersion relation can be rewritten as a set of sum rules for the negative moments of the difference of spectral functions,

$$
\frac{\mathrm{d}^{n}}{\left(\mathrm{~d} q^{2}\right)^{n}}\left(\Pi_{33}-\Pi_{88}\right)(0)=n ! \int_{s_{0}}^{\infty} d s \frac{\left(\rho_{\mathrm{V} 33}-\rho_{\mathrm{V} 88}\right)(s)}{s^{n+1}} \quad(n \geq 0) .
$$

In this form, the relevance of calculating $\Pi_{a a}\left(q^{2}\right)$ at low $q^{2}$ is evident.

Armed with the two-loop results obtained earlier in this paper, we can express the sum rules of Eq. (81) in terms of low energy constants of the effective chiral lagrangian. For instance, we have for the cases $n=0,1$,

$$
\begin{aligned}
\int_{s_{0}}^{\infty} & d s \frac{\left(\rho_{\mathrm{V} 33}-\rho_{\mathrm{V} 88}\right)(s)}{s}=\frac{1}{48 \pi^{2}} \ln \frac{M_{K}^{2}}{M_{\pi}^{2}} \\
& -\frac{4 M_{\pi}^{2}}{F_{0}^{2}} \cdot \frac{L_{9}^{(0)}\left(M_{K}^{2}\right)+L_{10}^{(0)}\left(M_{K}^{2}\right)}{8 \pi^{2}} \ln \frac{M_{K}^{2}}{M_{\pi}^{2}}+\frac{16\left(M_{K}^{2}-M_{\pi}^{2}\right)}{3 F_{0}^{2}} Q\left(M_{K}^{2}\right)(82)
\end{aligned}
$$

and

$$
\begin{aligned}
\int_{s_{0}}^{\infty} d s & \frac{\left(\rho_{\mathrm{V} 33}-\rho_{\mathrm{V} 88}\right)(s)}{s^{2}}=\frac{1}{480 \pi^{2}}\left(\frac{1}{M_{\pi}^{2}}-\frac{1}{M_{K}^{2}}\right) \\
& -\frac{1}{18 F_{0}^{2}}\left(\frac{1}{16 \pi^{2}}\right)^{2}\left(3-\ln \frac{M_{K}^{2}}{M_{\pi}^{2}}\right) \ln \frac{M_{K}^{2}}{M_{\pi}^{2}}+\frac{L_{9}^{(0)}\left(M_{K}^{2}\right)}{12 \pi^{2} F_{0}^{2}} \ln \frac{M_{K}^{2}}{M_{\pi}^{2}} .
\end{aligned}
$$

The sum rule in Eq. (82) has already been obtained by Knecht, Moussallam and Stern.20 However, since these authors calculate in generalized ChPT 
up to $\mathcal{O}\left(q^{5}\right)$, only part of the symmetry breaking term proportional to $Q$ appears on the RHS of their sum rule, i.e. they necessarily lack the $L_{9}^{(-1)}$ and $L_{10}^{(-1)}$ dependence occurring the defining relation for $Q$ in Eq. (82). By contrast, our expression in Eq. (82) is correct to $\mathcal{O}\left(q^{6}\right)$ in standard ChPT, and up to this order it is the combination of counterterms $\frac{4}{3} K_{3}^{(0)}-L_{9}^{(-1)}-L_{10}^{(-1)}$ which occurs. We note in passing that in addition to the term proportional to $L_{9}^{(0)}+L_{10}^{(0)}$, there would also be genuine two-loop contributions if the low energy constants were evaluated at a scale different from $M_{K}^{2}$. Turning to the second sum rule, we see that it contains only one low energy constant, $L_{9}^{(0)}$. Although $L_{9}^{(0)}$ is known to rather high accuracy from the electromagnetic charge radius of the pion, 2 it is nevertheless true that the relation in Eq. (83) offers a nontrivial check on the determination of this quantity.

Our two-loop representation of $\Pi_{33}-\Pi_{88}$ allows one to calculate the leading and next-to-leading order contributions to the RHS for all sum rules defined by Eq. (81) if $n \geq 0$. The leading term comes from the one-loop expression and is determined entirely by the $\pi \pi$ and $K \bar{K}$ continuum terms. The next-to-leading order term gets contributions from both continuua and resonances, the latter being parametrized by low energy constants like $L_{9}^{(0)}$. It might well be that these contributions are numerically as important as the leading term since the relevant physical phenomenon enters the calculation first only at that level of approximation.

Thus far, we have considered only the set of chiral sum rules associated with the difference, $\Pi_{33}-\Pi_{88}$, of the polarization functions. We can also derive sum rules for the individual vacuum polarizations. According to the asymptotic behaviour given in Eq. (79), at least one subtraction is required to obtain convergent sum rules. Thus we can write

$$
\int_{s_{0}}^{\infty} d s \frac{\rho_{\mathrm{V} a a}(s)}{s^{n+1}}=\frac{\mathrm{d}^{n}}{\left(\mathrm{~d} q^{2}\right)^{n}} \Pi_{a a}(0) \quad(a=3,8)
$$

for $n \geq 1$. As an example, we give the explicit expression for the isospin vacuum polarization $a=3$ with $n=1$,

$$
\begin{aligned}
\int_{s_{0}}^{\infty} & d s \frac{\rho_{\mathrm{V} 33}(s)}{s^{2}}=\frac{1}{480 \pi^{2}}\left(\frac{1}{M_{\pi}^{2}}+\frac{1}{2 M_{K}^{2}}\right) \\
& +\frac{1}{8 F_{0}^{2}}\left(\frac{1}{16 \pi^{2}}\right)^{2}\left(1-\frac{2}{3} \ln \frac{M_{K}^{2}}{M_{\pi}^{2}}\right)^{2}-\frac{L_{9}^{(0)}\left(M_{K}^{2}\right)}{8 \pi^{2} F_{0}^{2}}\left(1-\frac{2}{3} \ln \frac{M_{K}^{2}}{M_{\pi}^{2}}\right) \\
& -\frac{1}{F_{0}^{2}} P\left(M_{K}^{2}\right) .
\end{aligned}
$$


This sum rule clearly serves to fix the $\mathcal{O}\left(q^{6}\right)$ renormalization constant $P$. Note that the $s^{-n}$ sum rules which involve just the individual spectral functions are more slowly convergent than those involving a flavor difference of spectral functions because for the latter the leading term in the asymptotic expansion cancels.

To analyze the sum rules of Eqs. (82)-(85), it will be necessary to evaluate integrals containing negative moments of the spectral functions. We shall relegate such studies to a forthcoming publication, where we will also attempt to give realistic error bars on our determinations. Here, it is sufficient to touch upon the general strategy for calculating such integrals. As in Ref. [7], it is natural to divide the region of integration into three parts: A) the threshold region, B) an intermediate energy region where experimental information on the spectral functions is available, and $\mathrm{C}$ ) the asymptotic region. The asymptotic behaviour of the spectral functions is calculated using the OPE. As such, we anticipate the contribution from Region $\mathrm{C}$ to be especially small for the flavor-difference sum rules since for these the OPE will be proportional to light quark masses. Region $\mathrm{B}$ is the most difficult one to treat because it is here that intricacies of data analysis enter. Finally, in Region A we can use the improved low energy expansion of the spectral functions obtained in this paper.

What will be the outcome of numerically analyzing the new chiral rules? The answer is that one thereby determines the combinations of counterterms, particularly those of $\mathcal{O}\left(q^{6}\right)$, appearing in them. By no means is this a trivial task, as the following example will demonstrate. For definiteness, we focus on the sum rule of Eq. (82) and rewrite it as

$$
\begin{aligned}
\int_{s_{0}}^{\infty} & d s \frac{\left(\rho_{\mathrm{V} 33}-\rho_{\mathrm{V} 88}\right)(s)}{s}-\frac{1}{48 \pi^{2}} \ln \frac{M_{K}^{2}}{M_{\pi}^{2}}= \\
& -\frac{4 M_{\pi}^{2}}{F_{0}^{2}} \cdot \frac{L_{9}^{(0)}\left(M_{K}^{2}\right)+L_{10}^{(0)}\left(M_{K}^{2}\right)}{8 \pi^{2}} \ln \frac{M_{K}^{2}}{M_{\pi}^{2}}+\frac{16\left(M_{K}^{2}-M_{\pi}^{2}\right)}{3 F_{0}^{2}} Q\left(M_{K}^{2}\right)(86)
\end{aligned}
$$

so that only terms proportional to the $\mathcal{O}\left(q^{4}\right)$ and $\mathcal{O}\left(q^{6}\right)$ low energy constants appear on the RHS. We see from Eq. (86) that these counterterms are fixed by the difference between a spectral integral and a logarithmic mass ratio. However, the latter term can itself be expressed as a kind of spectral integral by employing the leading-orderf chiral representations for the isospin and hypercharge spectral functions. To demonstrate this, let us

\footnotetext{
${ }^{4}$ Similar arguments apply to next-to-leading order, but the corresponding expressions are much more complicated.
} 
first define energy scales $\Lambda_{3}$ and $\Lambda_{8}$ below which the leading-order chiral representations $\rho_{\mathrm{V} 33}^{\text {(chir) }}$ and $\rho_{\mathrm{V} 88}^{\text {(chir) }}$ are reasonable approximations to the physical spectral functions. We then carry out the integration to obtain

$$
\int_{4 M_{\pi}^{2}}^{\Lambda_{3}} d s \frac{\rho_{\mathrm{V} 33}^{(\mathrm{chir})}(s)}{s}-\int_{4 M_{K}^{2}}^{\Lambda_{8}} d s \frac{\rho_{\mathrm{V} 88}^{(\mathrm{chir})}(s)}{s} \doteq \frac{1}{48 \pi^{2}}\left[I\left(\frac{4 M_{\pi}^{2}}{\Lambda_{3}}\right)-I\left(\frac{4 M_{K}^{2}}{\Lambda_{8}}\right)\right],
$$

where the function $I\left(s_{0} / \Lambda\right)$ is given by

$$
\begin{aligned}
& I\left(\frac{s_{0}}{\Lambda}\right) \equiv \int_{s_{0}}^{\Lambda} \frac{d s}{s}\left[1-\frac{s_{0}}{s}\right]^{3 / 2} \\
& \quad=-\frac{2}{3}\left[1-\frac{s_{0}}{\Lambda}\right]^{3 / 2}-2\left[1-\frac{s_{0}}{\Lambda}\right]^{1 / 2}-\ln \frac{s_{0}}{\Lambda}+2 \ln \left[1+\left(1-\frac{s_{0}}{\Lambda}\right)^{1 / 2}\right]
\end{aligned}
$$

Upon removing the cutoffs in an appropriate manner, one regains the logarithmic term appearing on the LHS in Eq. (86), viz.

$$
\lim _{\substack{\Lambda_{3,8} \rightarrow \infty \\ \Lambda_{3} \rightarrow \Lambda_{8}}}\left[I\left(\frac{4 M_{\pi}^{2}}{\Lambda_{3}}\right)-I\left(\frac{4 M_{K}^{2}}{\Lambda_{8}}\right)\right]=\ln \left(\frac{M_{K}^{2}}{M_{\pi}^{2}}\right) .
$$

The point of this exercise is to stress that determination of the counterterms will involve spectral integrals over $\Lambda \leq s<\infty$ of the difference $\rho_{\mathrm{V}}^{\text {(phys) }}-\rho_{\mathrm{V}}^{(\text {chir })}$. The reader should appreciate the irony that, although negative moments enhance the threshold region in the new chiral sum rules, this has little influence on the counterterm determination. That is, as just shown the threshold region, in which $\rho_{\mathrm{V}}^{(\mathrm{phys})} \simeq \rho_{\mathrm{V}}^{(\mathrm{chir})}$, is suppressed via cancelation. Thus, to correctly infer values for the counterterms one must carefully analyze regions in which $\rho_{\mathrm{V}}^{\text {(phys) }} \neq \rho_{\mathrm{V}}^{\text {(chir) }}$, most crucially the intermediate energy region where available data fix the spectral functions.

Finally, let us point out that our discussion of Eq. (86) has yielded yet another insight. If one passes to the limit of, say, $S U(2)$ chiral symmetry, then the explicit logarithmic dependence on $M_{\pi}^{2}$ in Eq. (86) leads to a divergence. In order to maintain consistency, then so too must the spectral integral in Eq. (86) diverge. The discussion just given shows precisely, via the chiral representations for the spectral functions, how this comes about. Thus the explicit logarithmic dependence in the chiral sum rules correctly reflects the leading implicit infrared singularities of the spectral integrals which appear therein. 


\section{Conclusions}

We have used ChPT to determine the two vector current propagators $\Pi_{33}$ and $\Pi_{88}$ through two-loop order. The main results appear in Eq. (71) and Eq. (73). As expected, one finds the analytic structure corresponding to both $\pi \pi$ and $K \bar{K}$ thresholds for $\Pi_{33}$ and just the $K \bar{K}$ threshold for $\Pi_{88}$. There is no $3 \pi$ threshold for the propagators because we do not consider anomalous currents in this paper. The final results are seen to contain a total of six renormalization constants and this warrants some discussion. 24 The presence of the $\mathcal{O}\left(q^{4}\right)$ counterterms $L_{10}^{(0)}$ and $H_{1}^{(0)}$ already follows from the original one-loop analysis of Gasser and Leutwyler, whereas the remaining four arise from two-loop effects. Although the quantity $L_{9}^{(0)}$ is known from the one-loop analysis of the pion form factor, the other three $(P, Q, R)$ are new. As we have seen, all but $L_{9}^{(0)}$ contribute only to the real parts of the isospin and hypercharge propagators. The presence of these new renormalization constants is, of course, no surprise. For an effective theory like ChPT, the number of terms in the lagrangian increases sharply with loop order, and the renormalization constants are simply the set of quantities needed to render the theory meaningful at a given order. We shall comment shortly on how to partially constrain the new renormalization constants.

There are a number of interesting applications of the work described here. Of these, the most immediate involves the comparison between data and the chiral prediction for the isospin spectral function $\rho_{\mathrm{V} 33}\left(q^{2}\right)$. Our calculation allows extraction of the isospin and hypercharge spectral functions at nextto-leading order in the chiral expansion. For $\rho_{\mathrm{V} 33}\left(q^{2}\right)$ the correction turns out to be substantial even at energies very close to the two-pion threshold, $c f$. Fig. 5. From this figure we can see that our two-loop representation of the isospin spectral function is valid up to an energy of about $400 \mathrm{MeV}$.

Mention was made above of the counterterms which contribute to our final results. Three of these are new in the sense that they lie outside the phenomenology already established for the one-loop sector. They all occur in the real parts of the isospin and hypercharge propagators. By exploiting the asymptotic behaviour of the vector polarization functions $\Pi_{33}$ and $\Pi_{88}$ as inferred from the operator product expansion, we have derived a set of new chiral sum rules. Among these is a sum rule for the $\mathcal{O}\left(q^{4}\right)$ counterterm $L_{9}^{(0)}$. Since this quantity is already well-known, the sum rule offers a nontrivial check on the theory. The other sum rules fix $\mathcal{O}\left(q^{6}\right)$ counterterms, some of which also appear in the two-loop analysis of the process $\gamma \gamma \rightarrow \pi^{0} \pi^{0}$. 
We have begun the program of study outlined in Sect. 7 to constrain these quantities in terms of data, and results will be announced elsewhere.

Finally, work is well underway on a two-loop determination of the $S U(3)$ isospin and hypercharge axialvector current propagators. This study is a natural extension of both the present one and also of previous work done on the generalized Weinberg sum rules. (1] Although a chiral analysis of the axialvector propagators will superficially resemble that of the vector propagators, the two turn out to be very different in practice. In particular, a great deal of work on the renormalization of masses and decay constants of pseudoscalar mesons is required for the latter. Thus, we shall treat the axialvector sector separately in a forthcoming publication.

\section{Acknowledgements}

The research described in this paper was supported in part by the National Science Foundation and by Schweizerischer Nationalfonds. One of us (E.G.) wishes to acknowledge the kind hospitality of the theory group at the Rutherford Appleton Laboratory, where part of the work described herein was performed.

\section{References}

[1] J. Gasser and H. Leutwyler, Ann. Phys. 158 (1984) 142.

[2] J. Gasser and H. Leutwyler, Nucl. Phys. B250 (1985) 465.

[3] J. Stern, H. Sazdjian and N.H. Fuchs, Phys. Rev. D47 (1993) 3814; M. Knecht and J. Stern, 'Generalized Chiral Perturbation Theory', preprint IPNO/TH 94-53 (to appear in the second edition of the Daథne Physics Handbook, Eds: L. Maiani, G. Pancheri and N. Paver, INFN, Frascati).

[4] T. Das, V. Mathur and S. Okubo, Phys. Rev. Lett. 19 (1967) 859; J. Gasser and H. Leutwyler, Ref. [2]; G. Ecker, J. Gasser, A. Pich and E. de Rafael, Nucl. Phys. B321 (1989) 311.

[5] S. Weinberg, Phys. Rev. Lett. 18 (1967) 507.

[6] T. Das, G.S. Guralnik, V.S. Mathur, F.E. Low and J.E. Young, Phys. Rev. Lett. 18 (1967) 759. 
[7] J.F. Donoghue and E. Golowich, Phys. Rev. D49 (1994) 1513.

[8] We shall show in Appendix B that one can also use the one-loop pion form factor to obtain this information. Thus one has an independent check for the correctness of our two-loop analysis.

[9] We adopt the normalization of currents and decay constants appearing in J.F. Donoghue, E. Golowich and B.R. Holstein, Dynamics of the Standard Model, Cambridge University Press (1992).

[10] We work with covariantly defined T-products.

[11] G. Ecker, private communication.

[12] Our unrenormalized $S U(3)$ polarization functions $\Pi_{33}$ and $\Pi_{88}$ are consistent with the photonic polarization function appearing in B. Holdom, R. Lewis and R.R. Mendel, Z. Phys. C63 (1994) 71.

[13] S. Weinberg, Physica 96 A (1979) 327.

[14] G. Ecker, J. Gasser, A. Pich and E. de Rafael, Nucl. Phys. B321 (1989) 311.

[15] G. Ecker, J. Kambor and D. Wyler, Nucl. Phys. B394 (1993) 101.

[16] S. Coleman, J. Wess and B. Zumino, Phys. Rev. 177 (1969) 2239.

[17] C.G. Callan, S. Coleman, J. Wess and B. Zumino, Phys. Rev. 177 (1969) 2247.

[18] H.W. Fearing and S. Scherer, 'Extension of the Chiral Perturbation Theory Meson Lagrangian to Order $p^{6}$, TRIUMF preprint TRI-PP94-68 (1994).

[19] S. Bellucci, J. Gasser and M.E. Sainio, Nucl. Phys. B423 (1994) 80.

[20] M. Knecht, B. Moussallam and J. Stern, Nucl. Phys. B429 (1994) 125.

[21] M. Knecht, private communication.

[22] M.A. Shifman, A.I. Vainshtein and V.I Zakharov, Nucl. Phys. B147 (1979) 385.

[23] E. Braaten, S. Narison and A. Pich, Nucl. Phys. B373 (1992) 581. 
[24] We remind the reader that we have chosen to fix all such constants at a common renormalization energy scale, $M_{K}^{2}$.

[25] J. Gasser and H. Leutwyler, Nucl. Phys. B250 (1985) 517. 


\section{Appendix A: Feynman Integrals}

In this Appendix, we shall collect together definitions and properties of functions which play a central role in our analysis. In each case, one begins with an integral defined in $d$-dimensions and proceeds with its evaluation by isolating any divergence it might contain and determining the associated finite part.

\section{The Integral A}

The simplest such quantity is the scalar integral

$$
A\left(m^{2}\right) \equiv \int \frac{d^{d} k}{(2 \pi)^{d}} \frac{1}{k^{2}-m^{2}}
$$

The evaluation procedure is standard and we obtain

$$
\begin{aligned}
A\left(m^{2}\right) & =\frac{-i}{(4 \pi)^{d / 2}} \frac{\mu^{4-d}}{\mu^{4-d}} \Gamma\left(1-\frac{d}{2}\right)\left(m^{2}\right)^{(d-2) / 2} \\
& =\mu^{d-4}\left[-2 i m^{2} \bar{\lambda}-\frac{i m^{2}}{16 \pi^{2}} \log \left(\frac{m^{2}}{\mu^{2}}\right)+\ldots\right]
\end{aligned}
$$

where

$$
\lambda=\mu^{d-4} \bar{\lambda}=\frac{\mu^{d-4}}{16 \pi^{2}}\left[\frac{1}{d-4}-\frac{1}{2}(\log 4 \pi-\gamma+1)\right] .
$$

The quantity $\mu$, introduced in Eq. (91), is the mass scale which enters the calculation via the use of dimensional regularization. The $\mu^{d-4}$ prefactor in Eq. (92) ensures that $A\left(\mathrm{~m}^{2}\right)$ has the proper units in $d$-dimensions.

\section{The Integrals $\mathbf{B}, \mathbf{B}_{\mu}, \mathbf{B}_{\mu \nu}$}

Next we define

$$
\begin{aligned}
B_{\mu \nu}\left(q^{2}, m^{2}\right) & =\int \frac{d^{d} k}{(2 \pi)^{d}} \frac{k_{\mu} k_{\nu}}{\left(k^{2}-m^{2}\right) \cdot\left((q-k)^{2}-m^{2}\right)} \\
B_{\mu}\left(q^{2}, m^{2}\right) & =\int \frac{d^{d} k}{(2 \pi)^{d}} \frac{k_{\mu}}{\left(k^{2}-m^{2}\right) \cdot\left((q-k)^{2}-m^{2}\right)} \\
B\left(q^{2}, m^{2}\right) & =\int \frac{d^{d} k}{(2 \pi)^{d}} \frac{1}{\left(k^{2}-m^{2}\right) \cdot\left((q-k)^{2}-m^{2}\right)} .
\end{aligned}
$$


Since the scalar integral $B$ is divergent, it is often convenient to work with a function $\bar{B}$ made finite by subtraction,

$$
\bar{B}\left(q^{2}, m^{2}\right) \equiv B\left(q^{2}, m^{2}\right)-B\left(0, m^{2}\right),
$$

where, with the aid of Eq. (92), we have

$$
B\left(0, m^{2}\right)=\frac{\partial A\left(m^{2}\right)}{\partial m^{2}}=\frac{A\left(m^{2}\right)}{m^{2}}-\frac{i}{16 \pi^{2}} .
$$

The subtracted integral can be written as

$$
\begin{aligned}
\bar{B}\left(q^{2}, m^{2}\right) & =-\frac{i}{16 \pi^{2}} \int_{0}^{1} d x \log \left(1-x(1-x) \frac{q^{2}}{m^{2}}\right) \\
& =\frac{i}{96 \pi^{2}} \cdot \frac{q^{2}}{m^{2}}+\frac{i}{960 \pi^{2}} \cdot \frac{q^{4}}{m^{4}}+\ldots .
\end{aligned}
$$

To study $B_{\mu}$, we invoke covariance to write $B_{\mu}\left(q^{2}, m^{2}\right) \equiv B_{1} q_{\mu}$. Then, by considering $q^{\mu} B_{\mu}$ one finds $B_{1}=B / 2$ so that

$$
B_{\mu}\left(q^{2}, m^{2}\right)=\frac{q_{\mu}}{2} B\left(q^{2}, m^{2}\right) .
$$

The tensor integral $B_{\mu \nu}$ can be conveniently decomposed as

$$
B_{\mu \nu}\left(q^{2}, m^{2}\right) \equiv B_{21}\left(q^{2}, m^{2}\right) q_{\mu} q_{\nu}+B_{22}\left(q^{2}, m^{2}\right) g_{\mu \nu} .
$$

For notational simplicity, we shall temporarily suppress function arguments in the following. Study of the contraction $q^{\mu} B_{\mu \nu}$ leads to the relation

$$
q^{2} B_{21}+B_{22}=\frac{1}{2} A+\frac{q^{2}}{4} B
$$

whereas the trace $g^{\mu \nu} B_{\mu \nu}$ yields

$$
q^{2} B_{21}+d B_{22}=A+m^{2} B
$$

From these relations, it follows that

$$
\begin{aligned}
& B_{21}=\frac{1}{3}\left[\left(1-\frac{m^{2}}{q^{2}}\right) \bar{B}+\frac{A}{m^{2}}-\frac{5 i}{96 \pi^{2}}\right] \\
& B_{22}=-\frac{q^{2}}{12}\left[\left(1-\frac{4 m^{2}}{q^{2}}\right) \bar{B}+\frac{A}{m^{2}}\left(1-\frac{6 m^{2}}{q^{2}}\right)-\frac{i}{48 \pi^{2}}\right] .
\end{aligned}
$$


A combination of these integrals which appears naturally in loop diagrams is

$$
\begin{aligned}
T_{\mu \nu} & \equiv 4 B_{\mu \nu}+q_{\mu} q_{\nu} B-2 q_{\mu} B_{\nu}-2 q_{\nu} B_{\mu} \\
& =4 B_{\mu \nu}-q_{\mu} q_{\nu} B \\
& =\left(q_{\mu} q_{\nu}-g_{\mu \nu} q^{2}\right)\left(4 B_{21}-B\right)+g_{\mu \nu}\left(4 B_{22}+4 q^{2} B_{21}-q^{2} B\right),
\end{aligned}
$$

where we have introduced a convenient tensor decomposition for $T_{\mu \nu}$ in the last line. The relations in Eq. (104) can then be used to obtain

$$
T_{\mu \nu}=\left(q_{\mu} q_{\nu}-g_{\mu \nu} q^{2}\right)\left(4 \bar{B}_{21}+\frac{A}{3 m^{2}}\right)+2 A g_{\mu \nu}
$$

where we define the finite quantity $\bar{B}_{21}$ as

$$
\bar{B}_{21}\left(q^{2}, m^{2}\right) \equiv \frac{1}{12}\left[\left(1-\frac{4 m^{2}}{q^{2}}\right) \bar{B}-\frac{i}{48 \pi^{2}}\right] .
$$

\section{The Integrals $\mathrm{C}, \mathrm{C}_{\mu}, \mathrm{C}_{\mu \nu}$}

Finally we consider the set of integrals

$$
\begin{aligned}
C_{\mu \nu}\left(q^{2}, m^{2}\right) & =\int \frac{d^{d} k}{(2 \pi)^{d}} \frac{k_{\mu} k_{\nu}}{\left(k^{2}-m^{2}\right)^{2} \cdot\left((q-k)^{2}-m^{2}\right)}, \\
C_{\mu}\left(q^{2}, m^{2}\right) & =\int \frac{d^{d} k}{(2 \pi)^{d}} \frac{k_{\mu}}{\left(k^{2}-m^{2}\right)^{2} \cdot\left((q-k)^{2}-m^{2}\right)}, \\
C\left(q^{2}, m^{2}\right) & =\int \frac{d^{d} k}{(2 \pi)^{d}} \frac{1}{\left(k^{2}-m^{2}\right)^{2} \cdot\left((q-k)^{2}-m^{2}\right)} .
\end{aligned}
$$

We first use covariance to write the vector integral as $C_{\mu}=C_{1} q_{\mu}$. Analysis of the contracted form $q_{\mu} C^{\mu}$ then determines $C_{1}$ in terms of $C$,

$$
C_{1}\left(q^{2}, m^{2}\right)=\frac{1}{2 q^{2}}\left[q^{2} C\left(q^{2}, m^{2}\right)+\bar{B}\left(q^{2}, m^{2}\right)\right] .
$$

The scalar integral $C$ can itself be determined by beginning with the identity $q_{\mu} \partial B / \partial q_{\mu}=-2 q_{\mu} C^{\mu}$, from which it follows

$$
C\left(q^{2}, m^{2}\right)=\frac{1}{q^{2}-4 m^{2}}\left(-\bar{B}\left(q^{2}, m^{2}\right)+\frac{i}{8 \pi^{2}}\right) .
$$


Analogous to our treatment of the tensor integral $B_{\mu \nu}$, we write the quantity $C_{\mu \nu}$ as

$$
C_{\mu \nu}\left(q^{2}, m^{2}\right) \equiv C_{21}\left(q^{2}, m^{2}\right) q_{\mu} q_{\nu}+C_{22}\left(q^{2}, m^{2}\right) g_{\mu \nu} .
$$

As before, the contraction $q^{\mu} C_{\mu \nu}$ leads to one relation,

$$
q^{2} C_{21}+C_{22}=\frac{1}{4}\left[q^{2} C+B+\bar{B}\right],
$$

and the trace $g^{\mu \nu} C_{\mu \nu}$ yields another,

$$
q^{2} C_{21}+d C_{22}=B+m^{2} C .
$$

Finally we define a quantity $U_{\mu \nu}$ which, like $T_{\mu \nu}$, occurs in loop diagrams,

$$
\begin{aligned}
U_{\mu \nu} \equiv 4 C_{\mu \nu} & +q_{\mu} q_{\nu} C-2 q_{\mu} C_{\nu}-2 q_{\nu} C_{\mu} \\
=\left(q_{\mu} q_{\nu}\right. & \left.-g_{\mu \nu} q^{2}\right)\left(4 C_{21}-C-2 \frac{\bar{B}}{q^{2}}\right) \\
& \quad+g_{\mu \nu}\left(4 C_{22}+q^{2}\left[4 C_{21}-C-\frac{2 \bar{B}}{q^{2}}\right]\right) .
\end{aligned}
$$

With the input of the relations in Eqs. (114), (115), the final line in Eq. (116) results in the useful expression

$$
U_{\mu \nu}=-\left(q_{\mu} q_{\nu}-g_{\mu \nu} q^{2}\right) \frac{\bar{B}}{q^{2}}+g_{\mu \nu}\left(\frac{A}{m^{2}}-\frac{i}{16 \pi^{2}}\right) .
$$

\section{Appendix B: The Isospin Vector Spectral Function}

In the following, we shall show how to obtain an expression for the vectorcurrent spectral function good to two-loop order by using knowledge of the one-loop formulae for the pseudoscalar meson form factors. This will serve as a useful check of our two-loop analysis. For definiteness, we shall focus on the isospin spectral function.

We begin with the statement of unitarity for the isospin vector-current propagator. At sufficiently low energies, only the physical two-pion intermediate state contributes. We can use Eq. (15) of Ref. [7] and surrounding discussion to write

$$
\rho_{\mathrm{V} 33}(s)=\frac{1}{48 \pi^{2}}\left[1-\frac{4 M_{\pi}^{2}}{s}\right]^{3 / 2}\left|F_{\pi \pi}(s)\right|^{2} .
$$


If we interpret both sides of this equation in the context of a chiral expansion, then the spectral function to $(n+1)^{s t}$ order is expressible in terms of the pion form factor given to $n^{\text {th }}$ order. For example, in this way one immediately recovers the one-loop isospin spectral function of Ref. [1],

$$
\left.F_{\pi \pi}(s)\right|_{\text {tree }}=\left.1 \quad \Longrightarrow \quad \rho_{\mathrm{V} 33}(s)\right|_{1-\mathrm{loop}}=\frac{1}{48 \pi^{2}}\left[1-\frac{4 M_{\pi}^{2}}{s}\right]^{3 / 2}
$$

The corresponding prediction for the spectral function at two-loop order is obtained in like manner, although requiring more work. The chiral expansion of the pion form factor appears to first order in Ref. 25],

$$
F_{\pi \pi}(s)=1+2 H\left(s, M_{\pi}^{2}\right)+H\left(s, M_{K}^{2}\right)+\ldots,
$$

where, in terms of our notation,

$$
H\left(s, M^{2}\right) \equiv \frac{2 s}{3 F_{0}^{2}} L_{9}^{(0)}\left(M^{2}\right)-\frac{s}{F_{0}^{2}} i \bar{B}_{21}\left(s, M^{2}\right) .
$$

It then follows to two-loop order that

$$
\begin{aligned}
\rho_{\mathrm{V} 33}(s)= & \frac{1}{48 \pi^{2}}\left[1-\frac{4 M_{\pi}^{2}}{s}\right]^{3 / 2} \\
& \times\left(1+4 \operatorname{Re} H\left(s, M_{\pi}^{2}\right)+2 \operatorname{Re} H\left(s, M_{K}^{2}\right)\right)+\ldots \\
= & \frac{1}{48 \pi^{2}}\left[1-\frac{4 M_{\pi}^{2}}{s}\right]^{3 / 2}\left(1+\frac{2 s}{3 F_{0}^{2}}\left[4 L_{9}^{(0)}\left(M_{\pi}^{2}\right)+2 L_{9}^{(0)}\left(M_{K}^{2}\right)\right]\right. \\
& \left.-\frac{s}{F_{0}^{2}}\left[4 i \bar{B}_{21}\left(s, M_{\pi}^{2}\right)+2 i \bar{B}_{21}\left(s, M_{K}^{2}\right)\right]\right) .
\end{aligned}
$$

Upon noting that

$$
L_{9}^{(0)}\left(M_{\pi}^{2}\right)=L_{9}^{(0)}\left(M_{K}^{2}\right)+\frac{1}{128 \pi^{2}} \log \left(\frac{M_{K}^{2}}{M_{\pi}^{2}}\right),
$$

we obtain precisely the two-loop result of Eq. (76) derived earlier in this paper,

$$
\begin{aligned}
& \rho_{\mathrm{V} 33}(s)=\frac{1}{48 \pi^{2}}\left[1-\frac{4 M_{\pi}^{2}}{s}\right]^{3 / 2}\left[1+\frac{s}{F_{0}^{2}}\left[4 L_{9}^{(0)}\left(M_{K}^{2}\right)\right.\right. \\
& \left.\left.+\frac{1}{48 \pi^{2}} \log \left(\frac{M_{K}^{2}}{M_{\pi}^{2}}\right)-4 i \bar{B}_{21}\left(s, M_{\pi}^{2}\right)-2 i \bar{B}_{21}\left(s, M_{K}^{2}\right)\right]\right] .
\end{aligned}
$$




\section{Figure Captions}

Fig. 1 Comparison of 1-loop chiral prediction with data.

Fig. 2 Generic graph for computing vector-current propagators.

Fig. 3 One-loop graphs for $\Delta_{\mathrm{V} a b}^{\mu \nu}$.

Fig. 4 Two-loop graphs for $\Delta_{\mathrm{V} a b}^{\mu \nu}$.

Fig. 5 Comparison of 2-loop chiral prediction with data. The one-loop, two-loop and total contributions are denoted respectively by dotted, dashed and solid lines. 
This figure "fig1-1.png" is available in "png" format from: http://arxiv.org/ps/hep-ph/9501318v3 


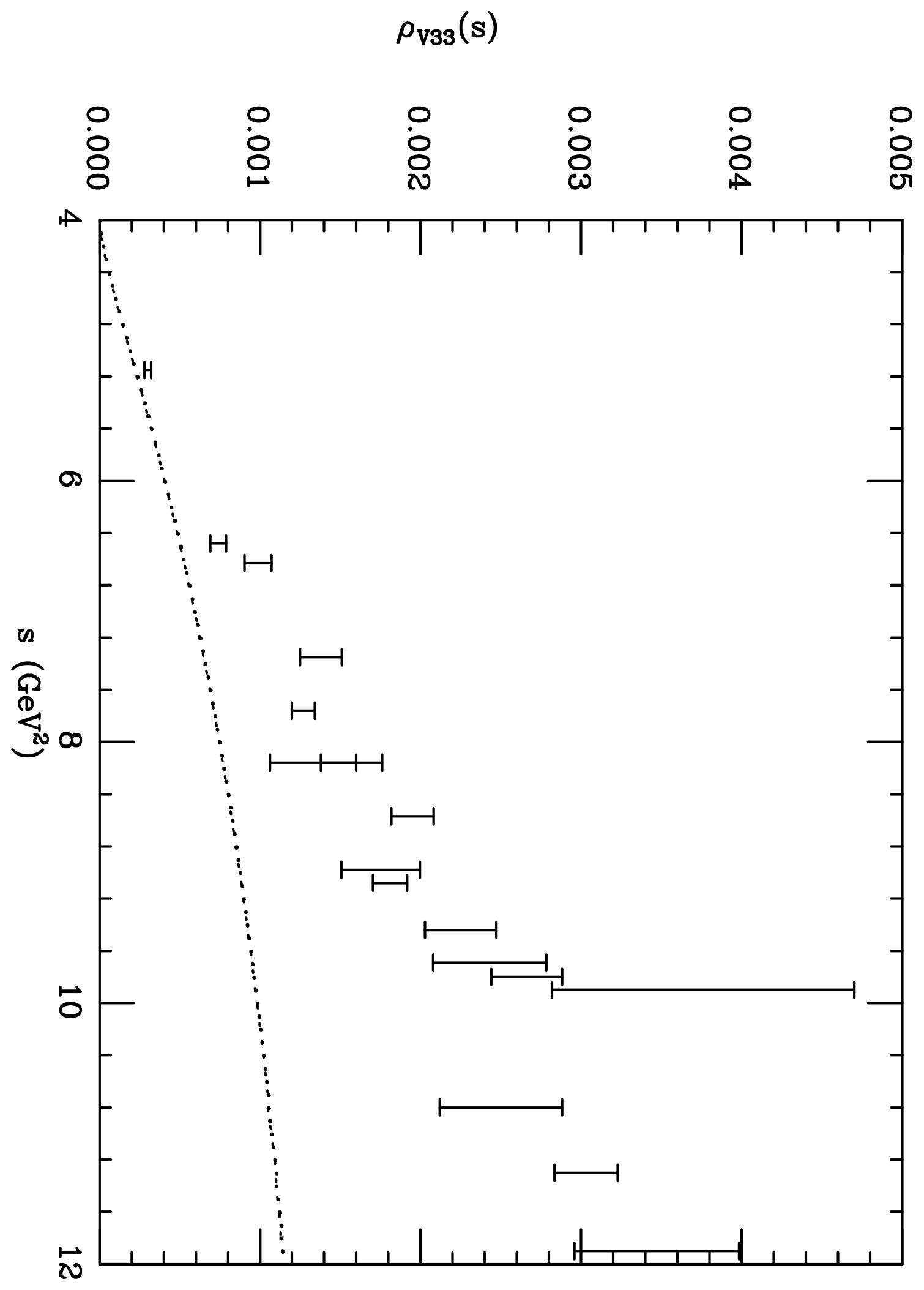


This figure "fig1-2.png" is available in "png" format from: http://arxiv.org/ps/hep-ph/9501318v3 
$\mathrm{q}, \mathrm{v}, \mathrm{b} \leadsto \mathrm{q}^{\prime}, \mu, \mathrm{a}$ 
This figure "fig1-3.png" is available in "png" format from: http://arxiv.org/ps/hep-ph/9501318v3 


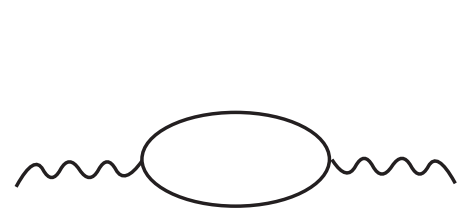

(a)

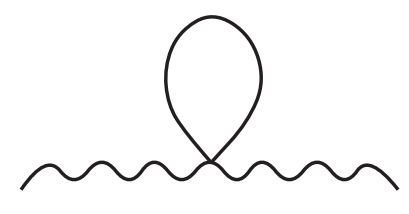

(b)

\section{$\sim \sim \sim \sim$}

(c)

One-loop diagrams 


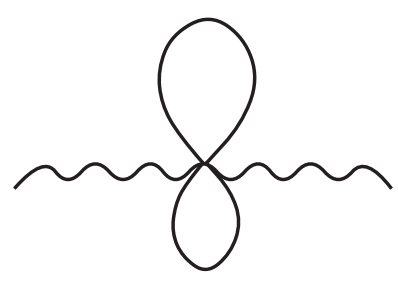

(a)

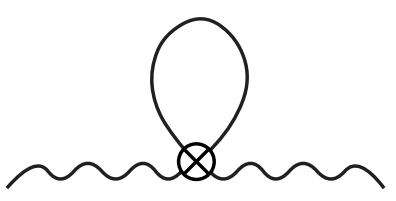

(d)

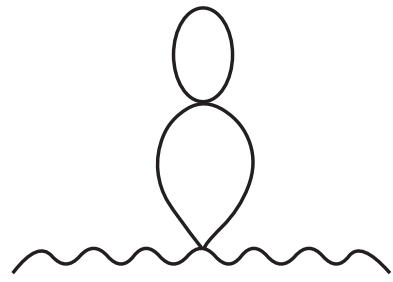

(b)

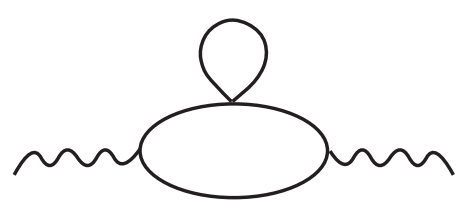

(e)

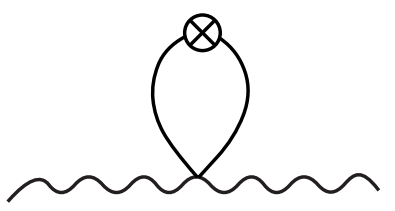

(c)

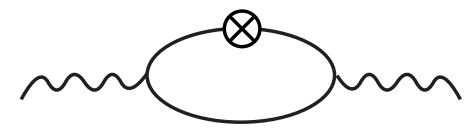

(f)

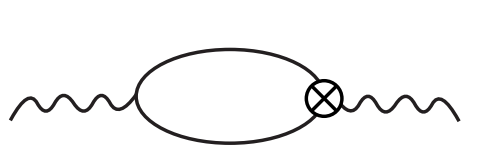

(g)

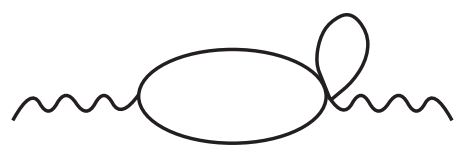

(h)

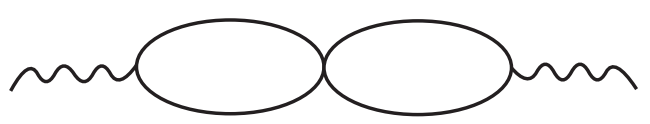

(i)

$\sim \sim \otimes \sim \sim$

(j)

Two-loop diagrams 


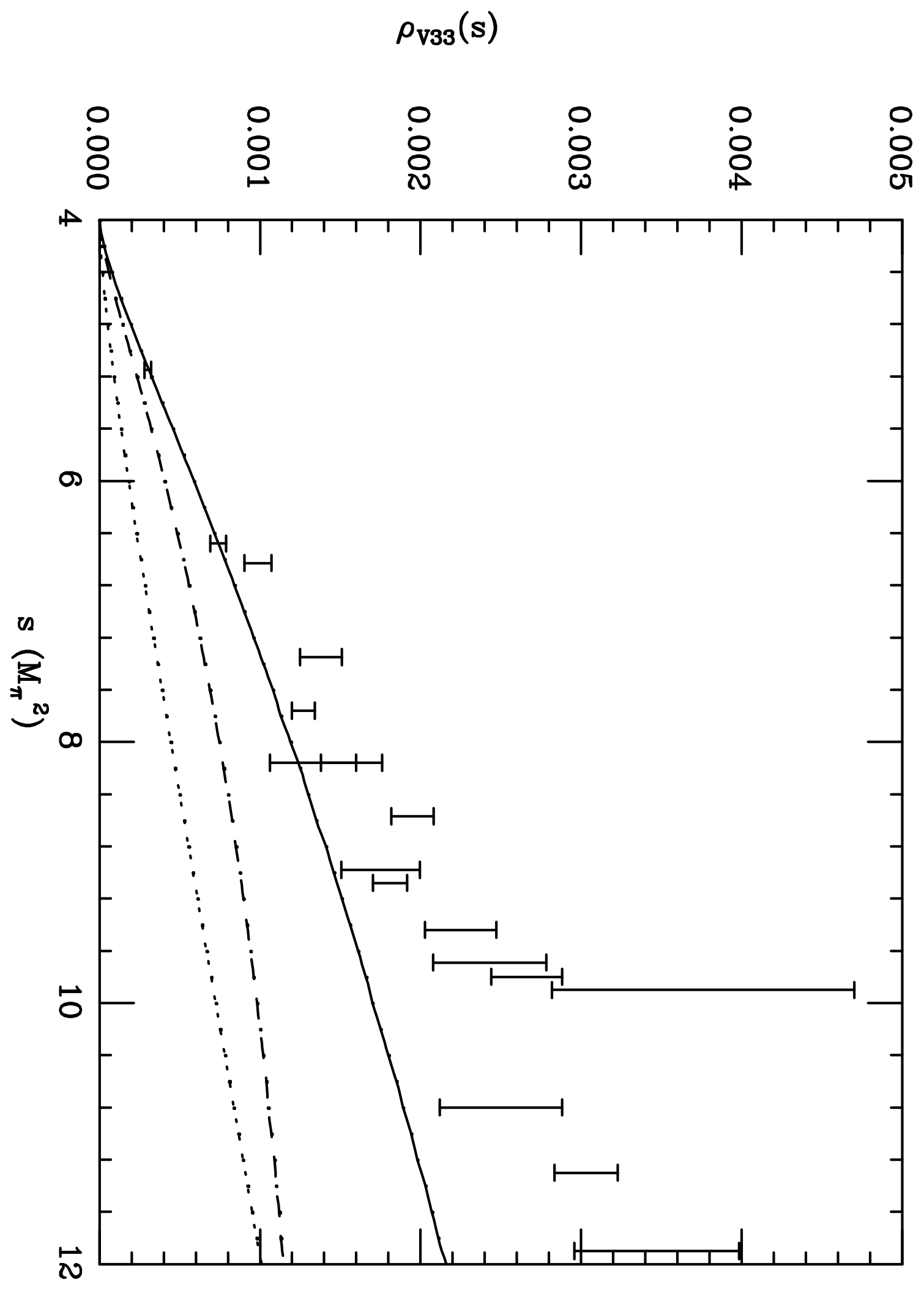

